ARTICLE

DOI: $10.1038 / s 41467-018-05745-w$

\title{
Nuclear-resident RIG-I senses viral replication inducing antiviral immunity
}

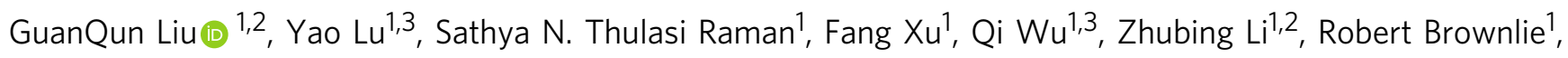
Qiang Liu (1) ${ }^{1,2,3}$ \& Yan Zhou (i) ${ }^{1,2,3}$

The nucleus represents a cellular compartment where the discrimination of self from non-self nucleic acids is vital. While emerging evidence establishes a nuclear non-self DNA sensing paradigm, the nuclear sensing of non-self RNA, such as that from nuclear-replicating RNA viruses, remains unexplored. Here, we report the identification of nuclear-resident RIG-I actively involved in nuclear viral RNA sensing. The nuclear RIG-I, along with its cytoplasmic counterpart, senses influenza A virus (IAV) nuclear replication leading to a cooperative induction of type I interferon response. Its activation signals through the canonical signaling axis and establishes an effective antiviral state restricting IAV replication. The exclusive signaling specificity conferred by nuclear RIG-I is reinforced by its inability to sense cytoplasmic-replicating Sendai virus and appreciable sensing of hepatitis B virus pregenomic RNA in the nucleus. These results refine the RNA sensing paradigm for nuclear-replicating viruses and reveal a previously unrecognized subcellular milieu for RIG-I-like receptor sensing.

\footnotetext{
${ }^{1}$ Vaccine and Infectious Disease Organization-International Vaccine Centre (VIDO-InterVac), University of Saskatchewan, Saskatoon, SK S7N 5E3, Canada.

${ }^{2}$ Vaccinology \& Immunotherapeutics Program, School of Public Health, University of Saskatchewan, Saskatoon, SK S7N 5E3, Canada. ${ }^{3}$ Department of Veterinary Microbiology, Western College of Veterinary Medicine, University of Saskatchewan, Saskatoon, SK S7N 5E3, Canada. Correspondence and requests for materials should be addressed to Y.Z. (email: yan.zhou@usask.ca)
} 
T he constant challenge of the vertebrate cells by invading pathogens drives the evolution of innate immune systems to rapidly detect and respond to non-self molecules, such as the virus-derived nucleic acids ${ }^{1}$. Depending on their intracellular localization, distinct germline-encoded pattern-recognition receptors (PRRs) engage specialized adapters to initiate immune signaling cascades from within different cellular compartments. To date, it has been well defined the roles of PRRs, including the Toll-like receptors, retinoic acid inducible gene I (RIG-I)-like receptors (RLRs), and cyclic GMP-AMP synthase (cGAS), in endosomal and cytosolic sensing of viral DNA and RNA ${ }^{2,3}$. However, the nuclear-replicating property of nearly all DNA viruses has shifted the PRR sensing paradigm toward the cell nucleus ${ }^{4}$. Emerging evidence delineates the functional roles of DNA sensors including IFI16 and cGAS in nuclear sensing of the Herpesviridae family, including Herpes simplex virus 1, human cytomegalovirus, Epstein-Barr virus, and Kaposi sarcomaassociated virus ${ }^{5-8}$. In contrast, most RNA viruses replicate within the cytoplasmic compartment. One of the primary RNA sensors, RIG-I, is well characterized as a cytosolic sensor of viral RNAs bearing $5^{\prime}$ diphosphates or triphosphates and juxtaposed short base-paired stretches ${ }^{9}$. Its activation initiates signaling cascades via the mitochondrial antiviral-signaling protein (MAVS), leading to the production of type I interferons (IFNs) which in turn upregulate other antiviral interferon-stimulated genes (ISGs) ${ }^{10,11}$. Interestingly, the members of the Orthomyxoviridae family represent a few RNA viruses replicating in the nuclear compartment ${ }^{12}$; however, the existence of a nuclear RNA sensing paradigm remains unexplored.

Apart from the IFN antagonism exerted by a plethora of virusencoded IFN antagonistic proteins targeting IFN induction and IFN signaling axes ${ }^{13,14}$, virus-mediated compartmentalization has recently become another immune evasion strategy. The members of the Flaviviridae family, including tick-borne encephalitis virus and hepatitis $\mathrm{C}$ virus, induce the formation of compartmentalized membrane structures to sterically segregate their viral agonists from PRRs ${ }^{15,16}$. This strategy conceals the viral replication site from the cytosolic RLRs, thereby minimizing the likelihood of non-self RNA sensing. Likewise, the nuclear replication of the Orthomyxoviridae family has also been hypothesized to act as an immune evasion strategy due in part to the cytoplasmic localization of classical RNA sensors such as RIG-I ${ }^{17}, 18$; however, this has never been experimentally substantiated. On the other hand, the host may have evolved a nuclear RNA sensing mechanism to counteract such immune evasion.

Influenza A virus (IAV) is the most-studied member of the Orthomyxoviridae family whose genome transcription and replication are closely associated with nuclear machinery ${ }^{19}$. It stimulates type I IFN expression in infected cells via a nearly strict RIG-I-dependent signaling cascade ${ }^{11,20}$. This RIG-I dependency underscores a long-standing question as to how the cytosolic RNA sensor RIG-I senses the nuclear replicating IAV. To date, the only RIG-I agonist characterized for influenza virus is the panhandle structure residing in either full-length or defectiveinterfering (DI) viral genomes ${ }^{21-23}$. We also reported that the IAV panhandle structure mediates and is mainly responsible for RIG-I activation and IFN induction in vitro ${ }^{24}$. Nonetheless, the spatiotemporal detection of the panhandle structure by RIG-I during the course of IAV infection remains unknown, particularly the accessibility of the ligands to RIG-I given the nuclear replication nature of IAV. Although an apparent interaction of cytoplasmic RIG-I with incoming viral ribonucleoprotein (vRNP) was visualized, no clear correlation with IFN induction was observed $^{25,26}$. Moreover, IAV differs from influenza B virus (IBV) in the kinetics of IFN induction; while IBV activates IFN signaling immediately after infection, IAV evades early recognition and induces IFN production at the late stage of infection ${ }^{27,28}$. Furthermore, IFN induction upon IAV infection could not be detected when viral RNA synthesis is inhibited ${ }^{29}$. These observations strongly suggest that RIG-I is capable of sensing IAV during the course of its nuclear replication, though the nature of the viral RNA species recognized remains unclear.

Here, we investigate the spatiotemporal activation of RIG-I in relation to IFN induction during IAV infection and refine the RIG-I sensing paradigm for IAV. We identify nuclear-resident RIG-I that is actively involved in sensing IAV replication in the nucleus, resulting in a cooperative IFN induction along with its cytoplasmic counterpart. Nuclear RIG-I initiates a MAVSdependent canonical signaling cascade, and is effective in establishing an antiviral state that restricts IAV infection. Furthermore, nuclear RIG-I remains inactive upon infection with cytoplasmicreplicating Sendai virus $(\mathrm{SeV})$, but exhibits signaling specificity toward nucleus-derived viral agonists, such as the pregenomic RNA (pgRNA) of hepatitis B virus (HBV). Our findings unravel a previously unrecognized nuclear pool of RIG-I sensing nuclear viral RNA and implicate a novel subcellular milieu for RLR sensing.

\section{Results}

Genuine presence of nuclear-resident RIG-I. RNA sensors are among the core vertebrate ISGs which exhibit very low basal expression but are highly upregulated by $\mathrm{IFN}^{30}$. In a focused exploration for the nuclear existence of the otherwise cytoplasmic RNA sensor RIG-I, we subjected A549 cells to SeV infection or IFN $\beta$ stimulation to globally upregulate RIG-I levels. The subcellular distribution of RIG-I was examined by immunofluorescence and subcellular fractionation. To ensure any observed RIG-I localization by immunofluorescence was genuine, RIG-I KO A549 cells were generated by CRISPR/Cas9-mediated genome editing (Supplementary Fig. 1) and the specificity of RIGI antibodies from different sources was carefully monitored by observing RIG-I staining in RIG-I KO A549 cells stimulated with $\mathrm{SeV}$ (Supplementary Fig. 2a). In the course of infection, $\mathrm{SeV}$ stimulated increasing amount of endogenous RIG-I expression, concomitant with increased levels of interferon regulatory factor 3 (IRF3) nuclear translocation (Fig. 1a and Supplementary Fig. 2b). Similarly, significant upregulation of RIG-I expression was observed in IFN $\beta$-primed cells (Supplementary Fig. 2b). Noticeably, a distinct nuclear staining of RIG-I was consistently observed, albeit at a much-reduced level compared with cytoplasmic RIG-I (Fig. 1a and Supplementary Fig. 2b). In THP-1 cells and HeLa cells ectopically expressing a FLAG-tagged RIG-I, nuclear RIG-I staining was also detected using either RIG-I antibody or a highly specific monoclonal antibody against the FLAG epitope (Supplementary Fig. 2c, d). To further authenticate the microscopic observation, SeV-infected or IFN $\beta$-primed A549 cells were subjected to cellular fractionation and the presence of nuclear RIG-I was consistently revealed (Fig. 1b, c, lanes 1-4). In addition, we examined whether the nuclear localization of RIG-I requires its downstream mitochondrial adapter MAVS. To this end, MAVS KO A549 cells were generated which could be efficiently primed by IFN $\beta$ for RIG-I upregulation (Fig. 1c, lanes 5-8), but were abolished in SeV-induced IRF3 phosphorylation and RIG-I upregulation due to a lack of MAVS-dependent IFN induction (Supplementary Fig. 2e). As with that in wild-type (WT) A549 cells, nuclear RIG-I was also detected in MAVS KO cells primed with IFN $\beta$ (Fig. 1c), demonstrating that the nuclear localization of RIG-I was independent of MAVS.

To further corroborate the presence of RIG-I in the nucleus, RIG-I was expressed with a FLAG tag together with a wellestablished nuclear export signal (NES) ${ }^{31}$. We asked whether the 
NES would redirect RIG-I out of the nucleus and negate the observed nuclear localization. To obviate potential interference from endogenous RIG-I, RIG-I KO A549 cells were complemented with stably maintained episomal constructs inducibly expressing a scrambled- (SLN-) or NES-tagged RIG-I. In response to doxycycline (Dox), these cells expressed high levels of respective RIG-I proteins (Fig. 1d). In contrast to SLN-RIG-I, which showed consistently detectable nuclear staining, NES-RIGI exhibited negligible nuclear localization (Fig. 1d). These cells were subsequently infected with IAV to examine whether virus infection induces RIG-I redistribution. Interestingly, IAV infection did not appear to change RIG-I localization, as both SLNRIG-I and NES-RIG-I exhibited the similar cellular distribution in infected cells compared to mock infection (Fig. 1d, e). Pearson's correlation coefficient analysis further confirmed a significantly greater colocalization of SLN-RIG-I with DAPI staining in the nucleus than that of NES-RIG-I (Fig. 1f). As a control, colocalization between nuclear IAV proteins and DAPI a

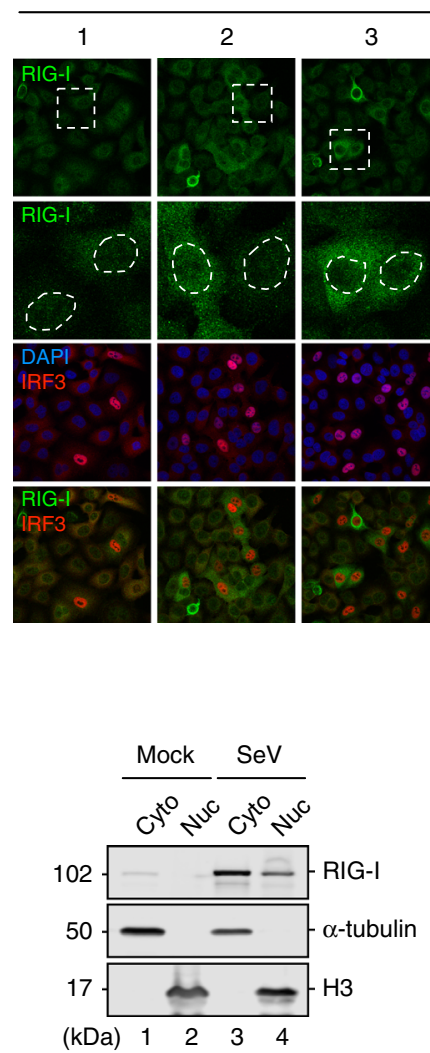

$\mathrm{SeV}$

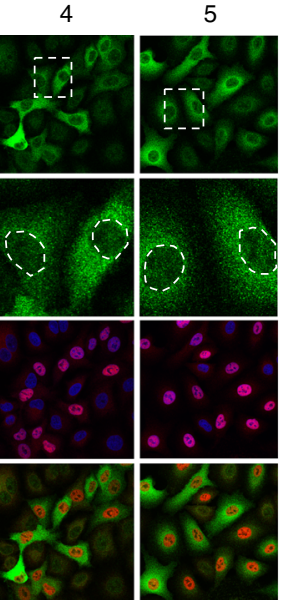

C

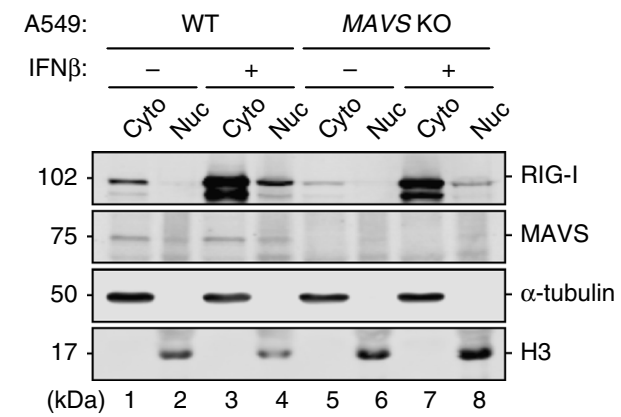

f Pearson's correlation

d

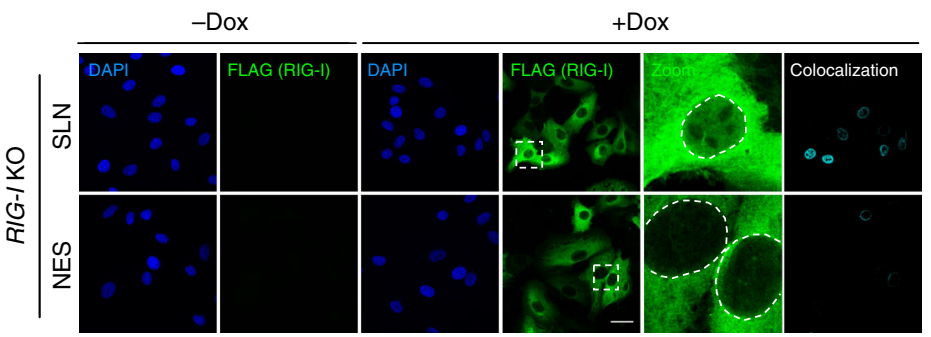
coefficients

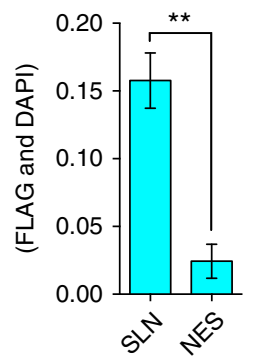

e

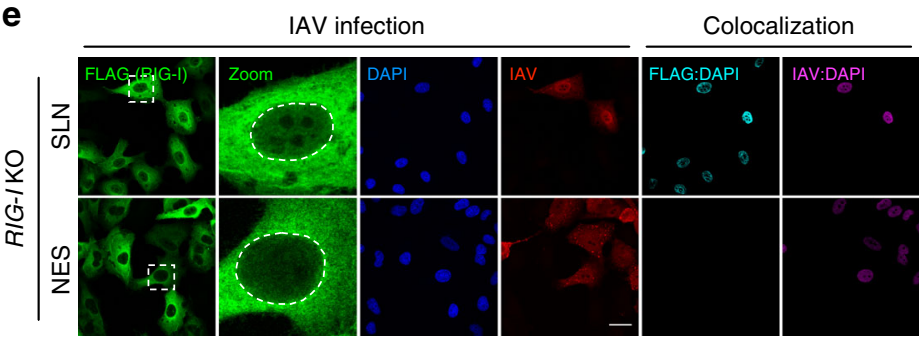

9

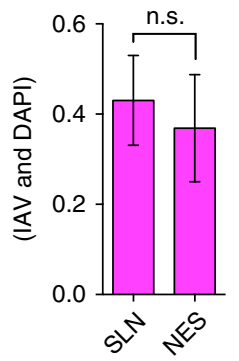


staining remained unchanged in infected cells expressing either SLN-RIG-I or NES-RIG-I (Fig. 1g). Collectively, these results demonstrated the genuine presence of RIG-I in the nucleus, which prompted us to further explore a plausible contribution of nuclear RIG-I to the sensing of nuclear viral RNA, particularly that of nuclear-replicating IAV.

Nuclear RIG-I constantly binds IAV vRNPs in the nucleus. RIG-I activation is generally inhibited during WT IAV infection unless the major IFN antagonistic protein NS1 is disrupted ${ }^{32,33}$. In contrast to that observed during $\mathrm{SeV}$ infection (Fig. 1a), endogenous RIG-I expression remained at basal levels in A549 cells infected with NS1-deficient IAV ( $\triangle \mathrm{NS} 1-\mathrm{ms})$ during a singlecycle infection (Fig. 2a). Upon prolonged infection, endogenous RIG-I levels remained low in the infected cells, whereas RIG-I upregulation was evident in non-infected neighboring cells, indicating an antiviral state primed by the IFN produced from the infected cells (Supplementary Fig. 3a). Since the low level of RIG-I in IAV-infected cells greatly hampered the tracking of its distribution by immunofluorescence, we sought to generate a recombinant mutant virus deficient of both NS1 and another viral protein $\mathrm{PA}-\mathrm{X}$ which poses a strong shutoff activity that globally supresses host protein expression ${ }^{34}$. We were able to rescue a double-deficient virus (PAfs $\triangle \mathrm{NS} 1$ ), whose PA and NS segments carry X-ORF frameshifting mutations and NS1-ORF deletion, respectively. PAfs $\triangle \mathrm{NS} 1$ was significantly attenuated in growth in IFN competent cells but replicated to a level that was two-log lower than the WT and $\triangle$ NS1-ms viruses in MDCK cells expressing NS1. Although no greater RIG-I upregulation was observed in infected cells during a single-cycle (Fig. 2b) or prolonged infection (Supplementary Fig. 3a), PAfs $\Delta$ NS1 stimulated IRF3 nuclear translocation as efficiently as $\Delta$ NS1-ms (Fig. 2a, b). Of note, IRF3 nuclear translocation was abolished in RIG-I KO A549 cells infected with either virus, demonstrating a strict activation of the RIG-I signaling pathway by both viruses (Supplementary Fig. 3b). Interestingly, a comparison between the two RIG-I-activating mutant viruses revealed distinct patterns of vRNP distribution. Using either fluorescence in situ hybridization (FISH) specifically detecting the M segment vRNA or the viral NP protein as a vRNP indicator, we observed substantial vRNP retention for PAfs $\triangle \mathrm{NS} 1$ in the nucleus during a single-cycle infection, whereas $\triangle$ NS1-ms exhibited vRNP distribution in both the nucleus and cytoplasm (Fig. 2a, b and Supplementary Fig. 3b). Despite such significant difference in vRNP localization, the kinetics of IRF3 activation induced by the two viruses resembled each other (Fig. 2c). Moreover, monitoring the vRNA localization in relation to IRF3 nuclear translocation revealed that RIG-I activation was closely associated with the nuclear vRNA accumulation; IRF3 activation was first detectable at 4 h.p.i and increased up to 8 h.p.i, concomitant with the course of nuclear vRNA accumulation of PAfs $\Delta$ NS1 (Fig. 2b). Taken together, that
PAfs $\triangle$ NS1 manifested impaired vRNP nuclear export yet stimulated IRF3 nuclear translocation as efficiently as $\Delta$ NS1-ms indicated a nuclear sensing event by the nuclear-resident RIG-I.

To sense nuclear viral RNA synthesis, the nuclear RIG-I would possibly interact with IAV vRNP to gain closer proximity to the immunostimulatory RNA species, such as the vRNA and cRNA $^{21,24}$. We performed cellular fractionation followed by coimmunoprecipitation to examine whether nuclear RIG-I associates with vRNPs. During WT or $\triangle$ NS1-ms infection, endogenous nuclear RIG-I interacted with vRNPs as efficiently as cytoplasmic RIG-I (Fig. 2d). Given the relative amount of immunoprecipitated proteins, nuclear RIG-I associated with even more vRNPs than the cytoplasmic RIG-I (Fig. 2d). Nuclear RIG-I association with vRNPs was likewise detected for seasonal H3N2 (A/Victoria/3/ 75) and avian H7N3 (A/chicken/British Columbia/CN-6/2004) viruses (Fig. 2e), ruling out a subtype-specific effect. To evaluate the relative temporal contribution of the two cellular pools of RIG-I to vRNP association, we monitored the extent of vRNP binding by cytoplasmic and nuclear RIG-I at early and late time points after WT virus infection. While cytoplasmic RIG-I associated with limited levels of vRNPs at 6 h.p.i, the interaction between nuclear RIG-I and vRNPs was noticeably higher (Fig. 2f, lane 3 vs. lane 6). At 14 h.p.i, the cytoplasmic RIG-I showed increased levels of vRNP association compared to that at 6 h.p.i (Fig. 2f, lane 3 vs. lane 9), while nuclear RIG-I manifested constant vRNP association (Fig. 2f, lane 6 vs. lane 12). These observations fit in the time frame of IAV life cycle during which the incoming vRNPs are rapidly imported into the nucleus where the nuclear RIG-I accounts for the early recognition. With the increased nuclear export of progeny vRNPs, both pools of RIG-I are involved in vRNP interaction at the late stage of infection. Collectively, these results demonstrated a sustained interaction of nuclear-resident RIG-I with vRNP in the IAV life cycle, particularly at early time points when the abundance of cytoplasmic RIG-I agonists is limited.

Nuclear-localized RIG-I senses nuclear viral RNA replication. To further scrutinize the functional role of nuclear RIG-I in RNA sensing, we took advantage of the IAV RNP reconstitution system, which recapitulates nuclear RNA synthesis with the minimal set of viral proteins ${ }^{35}$. Consistent with that in infected A549 cells (Fig. 2d), a nuclear pool of RIG-I was detected in RNP reconstituted $293 \mathrm{~T}$ cells ectopically expressing a FLAG-tagged RIG-I which also bound to nuclear vRNPs (Supplementary Fig. 4a). The RNA species extracted from the nuclear RIG-I immunoprecipitates were immunostimulatory when transfected into 293T reporter cells (Supplementary Fig. 4b). We next examined the cellular localization of viral RNA species in RNP reconstituted cells, particularly that of the essential RIG-I agonists (vRNA and cRNA) as previously reported ${ }^{21,24}$. While a portion of viral mRNA was exported to the cytoplasm, full-length vRNA and

Fig. 1 Genuine presence of RIG-I in the cell nucleus. a A549 cells were infected with Sendai virus (SeV) at $50 \mathrm{HAU} / \mathrm{mL}$. At indicated time points postinfection, cells were subjected to immunofluorescence for RIG-I (green) and IRF3 (red). Nuclei were stained with DAPI (blue). Boxed area was enlarged and the nuclear regions were outlined to highlight the nuclear RIG-I staining. Scale bar $=25 \mu \mathrm{m}$. b A549 cells were left uninfected, or infected with SeV $(50 \mathrm{HAU} / \mathrm{mL}$ ) for $16 \mathrm{~h}$. Cells were subjected to cellular fractionation and the presence of RIG-I in the cytoplasmic (Cyto) and nuclear (Nuc) fractions was determined by immunoblotting. $\alpha$-tubulin and histone $\mathrm{H} 3$ served as the markers for the cytoplasmic and nuclear fractions, respectively. c A549 WT or MAVS KO cells were left untreated, or primed with human IFN $\beta(500 \mathrm{U} / \mathrm{mL})$ for $16 \mathrm{~h}$. Cellular fractionation was performed as in $\mathbf{b}$, and the presence of RIGI and MAVS in both fractions was determined by immunoblotting. d, e A549 RIG-I KO cell lines inducibly expressing SLN-RIG-I or NES-RIG-I were left noninduced (-Dox), or induced with $1 \mu \mathrm{g} / \mathrm{mL}$ doxycycline (+Dox) for $4 \mathrm{~h}$ followed by mock (d) or WT PR8 infection $(\mathbf{e}, \mathrm{MOI}=5)$ for $14 \mathrm{~h}$. Cells were subjected to immunofluorescence for FLAG-RIG-I (green) and IAV (red). Nuclei were stained with DAPI (blue). Scale bar $=25 \mu \mathrm{m}$. Colocalization of FLAG/DAPI channels, and IAV/DAPI channels was analyzed using ImageJ and the colocalizing pixels were represented in cyan and magenta, respectively. f, $\mathbf{g}$ Pearson's correlation coefficients were calculated from at least three random fields with $\sim 20$ cells. Data are shown as mean \pm SD. Significant differences were determined by an unpaired Student's $t$-test. ${ }^{\star \star} p<0.01$; n.s. not significant 
a

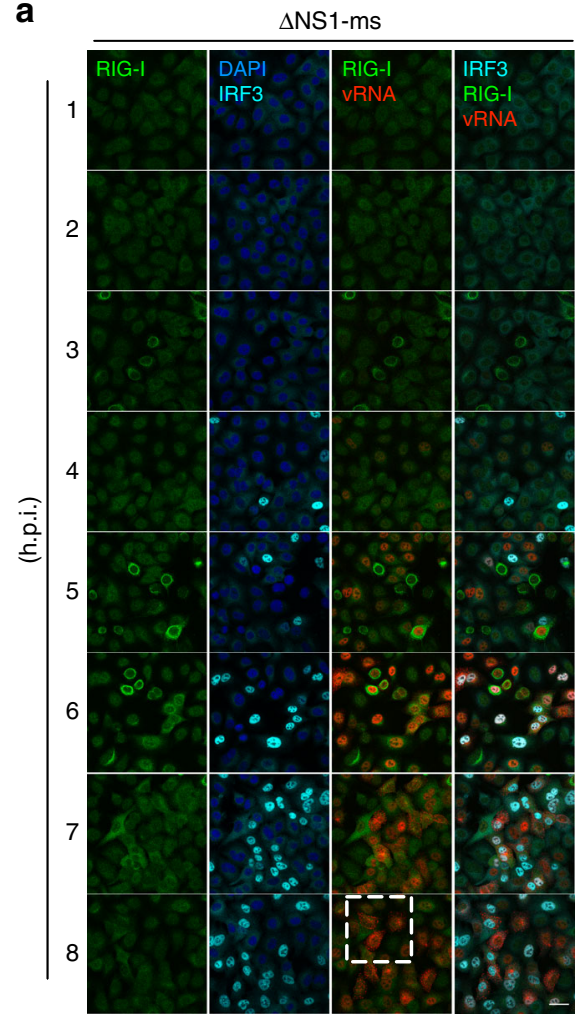

b

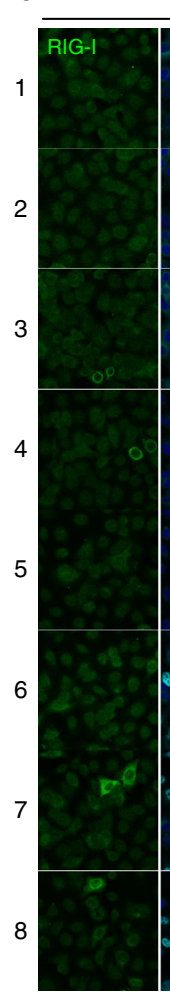

PAfs $\triangle N S 1$

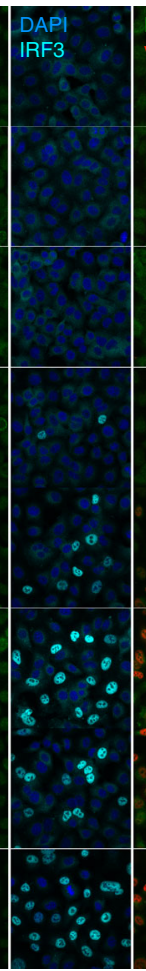

C
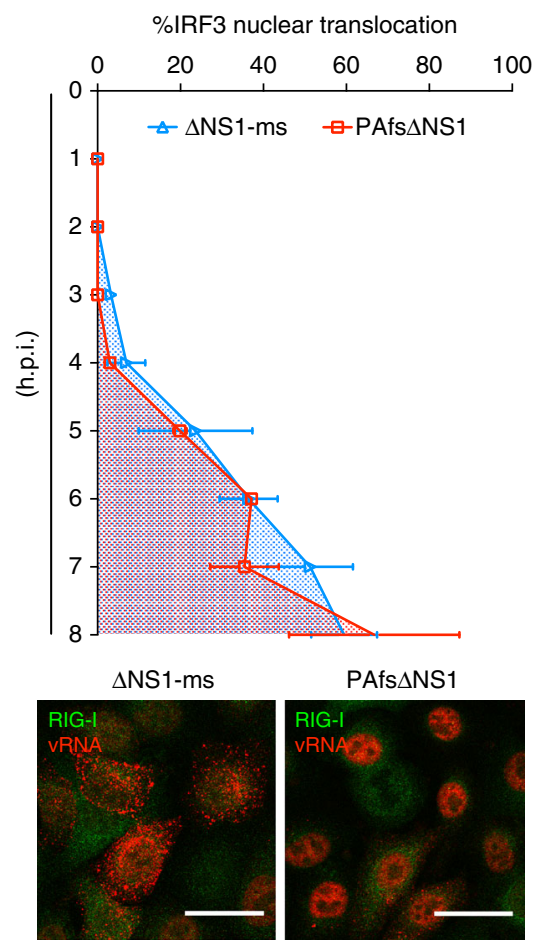

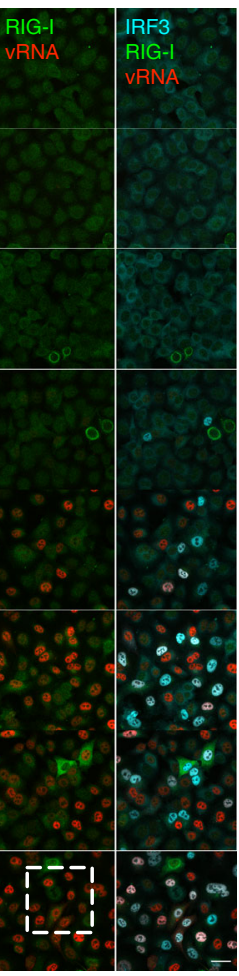

e

Vic75 (H3N2) BC04 (H7N3)
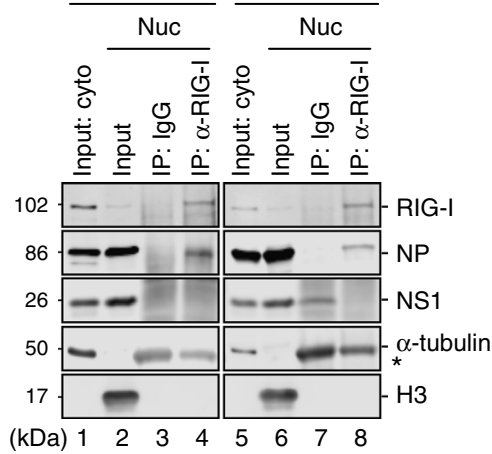

$\mathbf{f}$ $\frac{6 \mathrm{~h}}{\text { Cyto }} \frac{14 \mathrm{~h}}{-} \frac{\text { Nuc }}{-} \frac{\text { Nyto }}{-}$

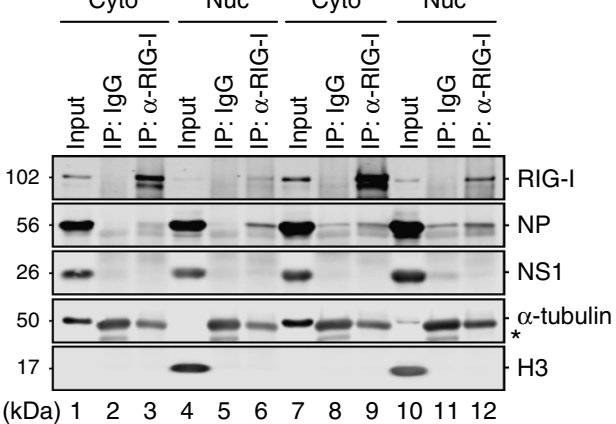

Fig. 2 Nuclear-resident RIG-I constantly binds IAV vRNPs in the nucleus. a, b A549 cells were infected with $\Delta$ NS1-ms or PAfs $\Delta N S 1$ virus at an MOI of 10. At indicated time points post-infection, cells were subjected to immunofluorescence for RIG-I (green) and IRF3 (cyan), and FISH for VRNA of the M segment (red). Nuclei were stained with DAPI (blue). The insets highlight the distinct vRNA distribution of the two mutant viruses. Scale bar $=25 \mu \mathrm{m}$. c Percentage of A549 cells with IRF3 nuclear translocation upon $\Delta$ NS1-ms and PAfs $\Delta$ NS1 infections was quantified from at least three random fields with $\sim 100$ cells for each time point after infection. $\mathbf{d}$ A549 cells were infected with WT PR8 or $\Delta$ NS1-ms $(\Delta)$ at an MOI of 5. At 8 h.p.i, cells were subjected to cellular fractionation and both cytoplasmic (Cyto) and nuclear (Nuc) fractions were immunoprecipitated (IP) with the RIG-I antibody or an IgG isotype control. The immunoprecipitates were analyzed by immunoblotting for RIG-I, NP, and NS1. $\alpha$-tubulin and histone H3 served as the markers for the cytoplasmic and nuclear fractions, respectively. The heavy chains of IP antibodies are indicated by an asterisk $\left(^{\star}\right)$. e Co-immunoprecipitation in the nuclear fractions of A549 cells infected with A/Victoria/3/75 (H3N2) or A/chicken/British Columbia/CN-6/2004 (H7N3) was performed as in (d). f A549 cells were infected with WT PR8 $(\mathrm{MOI}=2)$ for 6 or $14 \mathrm{~h}$. Cells were subjected to cellular fractionation and co-immunoprecipitation in both the cytoplasmic and nuclear fractions was performed as in (d)

cRNA of the NA segment were strictly confined to the nuclei of reconstituted cells (Fig. 3a). Reconstitution in the presence of a catalytically inactive PB1 (PB1a) served as the baseline for quantification of viral RNA levels ${ }^{36}$. Similarly, the FISH analysis revealed exclusive nuclear staining of $\mathrm{M}$ vRNA upon $\mathrm{M}$ segment reconstitution (Fig. 3b).

Accordingly, we specifically examined whether a nuclearlocalized RIG-I responds to IAV RNA synthesis leading to IFN induction. RIG-I was tagged with an SV40 large T antigen nuclear localization signal (NLS) to direct it to the nucleus (Fig. 3b).
While SLN-tagged and NES-tagged RIG-I responded efficiently to the cytoplasmic delivery of a synthetic RIG-I ligand, NLS-RIG-I remained inactive upon stimulation (Fig. 3c). Next, 293T cells were reconstituted with each of the eight viral segments in the presence of SLN-RIG-I, NLS-RIG-I, or GFP. None of these constructs affected IAV polymerase activity (Supplementary Fig. 5a). Although SLN-RIG-I overexpression resulted in a detectable residual level of IFN $\beta$ promoter activation, reconstitution of the PA, HA, NA, and M segments significantly enhanced IFN induction (Fig. 3d). In contrast, NLS-RIG-I itself showed 
minimal IFN induction but responded significantly to the reconstitution of most segments, except for the NP and NS segments (Fig. 3e). This IFN suppression by NP and NS reconstitutions was also observed in the presence of SLN-RIG-I (Fig. 3d). While the expression of NS1 conferred the inhibitory effect, NP protein alone did not impede dsRNA-induced IFN induction (Supplementary Fig. 5b). However, IFN $\beta$ promoter activation by NA reconstitution was suppressed by NP expression in a dose-dependent manner (Supplementary Fig. 5c), suggesting that NP level negatively regulates the generation of a

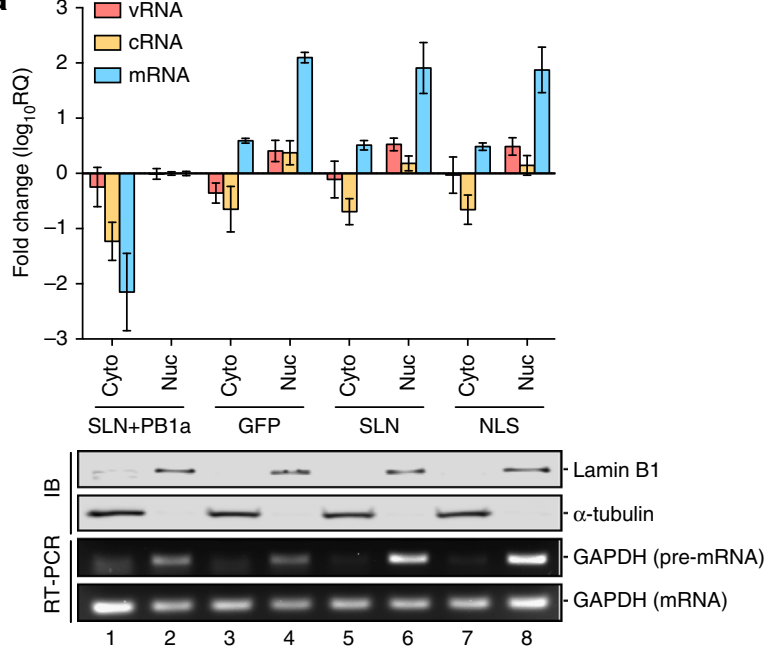

C
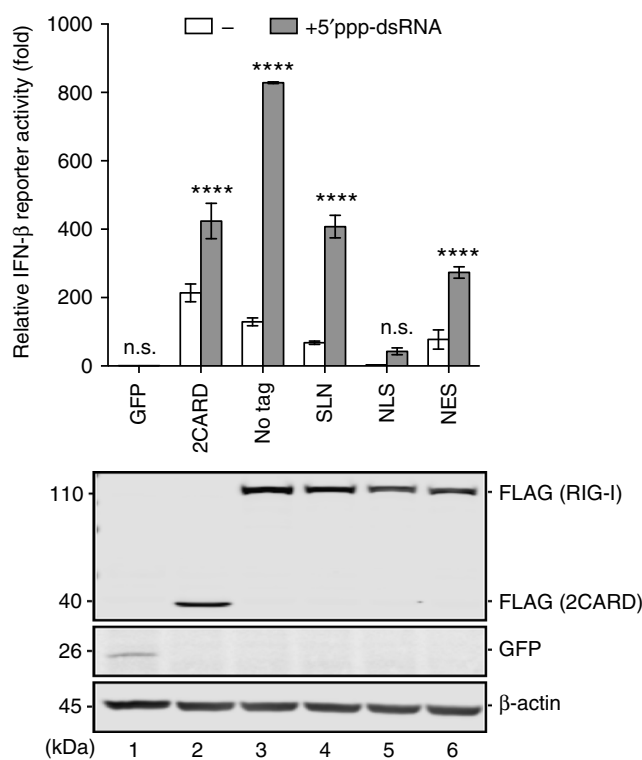

f

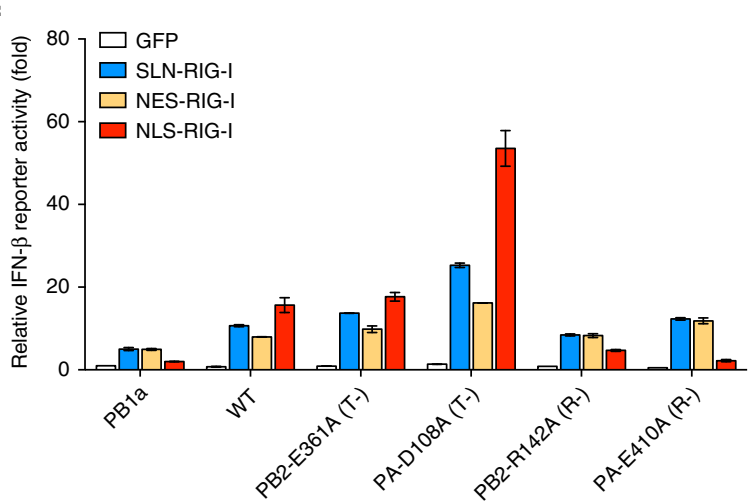

b
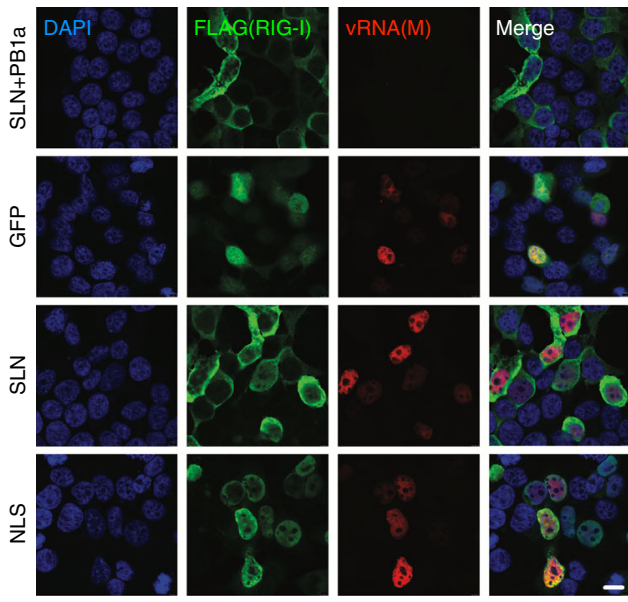

d

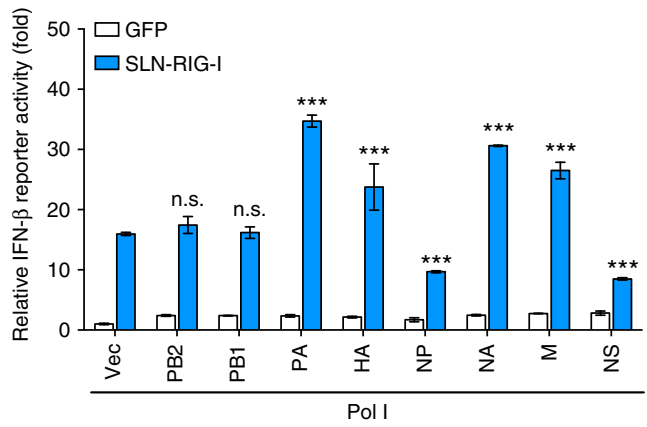

e
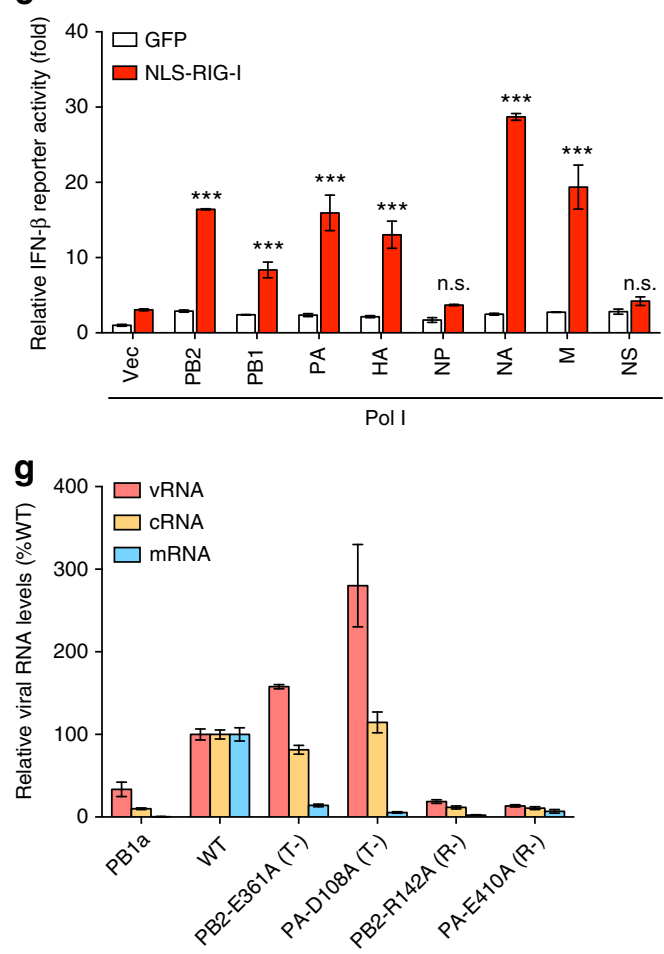
immunostimulatory RNA. Nonetheless, these results demonstrated that NLS-RIG-I was competent in sensing viral RNA synthesis in the nucleus.

Next, we examined the requirement of viral transcription and replication for NLS-RIG-I activation. Reconstitution in the presence of transcription-defective polymerases (PB2-E361A and PA-D108A) $)^{37,38}$ was able to generate viral RNA species stimulating NLS-RIG-I. In contrast, no apparent IFN $\beta$ promoter activation was detected for reconstitution with replicationdefective polymerases (PB2-R142A and PA-E410A) 39,40 (Fig. 3f). All mutant polymerases expressed to similar levels as the WT polymerase (Supplementary Fig. 6a). Quantification of viral RNA levels produced by mutant polymerases further revealed that the levels of IFN $\beta$ promoter activation were proportional to that of the full-length vRNA (Fig. 3f, g). This correlation was further confirmed by a dose-dependent activation of IFN $\beta$ promoter with increasing amount of vRNA-expressing plasmids (Supplementary Fig. 5d). Notably, compared to SLN-RIG-I and NES-RIG-I which consistently showed basal levels of activity, NLS-RIG-I exhibited superior signaling capacity in sensing augmented viral replication associated with transcription-defective polymerases (Fig. 3f, g). It is noted that no DI RNA was detected upon NA reconstitution (Supplementary Fig. 6b). Collectively, these results demonstrated that the nuclear-localized RIG-I senses nuclear viral RNA replication and is able to elicit greater IFN response possibly by gaining closer proximity to immunostimulatory viral RNA.

Nuclear RIG-I senses IAV via the canonical signaling axis. In the context of viral infection, we assessed the ability of nuclear RIG-I to sense IAV replication. RIG-I KO A549 cell lines inducibly expressing NLS-RIG-I and RIG-I with K270A mutation were constructed, with the latter serving as a signaling-inactive control $^{41}$. As the ectopic expression of RIG-I for an extended period of time elicits autonomous IFN response thereby inhibiting viral infection, we limited the Dox-induced RIG-I expression in inducible A549 cells to $4 \mathrm{~h}$. These cell lines, alongside with the SLN-RIG-I and NES-RIG-I-expressing cell lines, were subsequently infected with PAfs $\Delta \mathrm{NS} 1$ or $\triangle \mathrm{NS} 1-\mathrm{ms}$ and probed for IRF3 nuclear translocation. Consistent with that in RNPreconstituted 293T cells, complementation of RIG-I KO A549 cells with NLS-RIG-I mediated IRF3 nuclear translocation as efficiently as the SLN-RIG-I and NES-RIG-I, whereas K270ARIG-I did not respond to IAV infection (Fig. 4a). Although PAfs $\triangle N S 1$ exhibited confined vRNA to the nucleus, it elicited comparable levels of IRF3 activation to that of $\triangle N S 1-m s$ infection (Fig. 4a). Given the low levels of endogenous nuclear RIG-I in WT cells, we titrated Dox concentration on NLS-RIG-Iexpressing cells to match its expression comparable to the physiological levels (Supplementary Fig. 7a). Upon $\Delta$ NS1-ms infection, low levels of NLS-RIG-I expression mediated efficient IFN $\beta$ and IP10 induction in a Dox dose-dependent manner (Supplementary Fig. 7b, c). Strikingly, compared to RIG-I KO cells, even trace amount of NLS-RIG-I remained active in sensing IAV infection and inducing antiviral gene expression (Supplementary Fig. 7d-g). Next, we compared the kinetics of IRF3 activation mediated by compartment-specific RIG-I in response to PAfs $\triangle$ NS1 virus infection. Although IRF3 activation in cells expressing either RIG-I peaked at 7 h.p.i, both NES-RIG-I and NLS-RIG-I-expressing cell lines exhibited delayed IRF3 activation during 4 to 6 h.p.i (Fig. 4 b, c), suggesting cooperative roles of cytoplasmic and nuclear RIG-I in sensing IAV replication.

We next delineated the signaling cascade involved in nuclear RIG-I sensing. In RNP reconstitution, we validated that NLSRIG-I activation as determined by IFN $\beta$ promoter activation correlated with IRF3 phosphorylation and expression of antiviral genes such as IFN $\beta$ and IP10 (Fig. $4 \mathrm{~d}$ and Supplementary Fig. 8). Point mutations that abrogated three well-characterized RIG-I signaling motifs were introduced into NLS-RIG-I ${ }^{41-44}$. NA reconstitution in the presence of NLS-RIG-I with abolished ATPase activity (K270A), tripartite motif-containing protein 25 (TRIM25) interaction (T55I), or RNA binding (K888E) failed to induce IFN $\beta$ promoter activation (Fig. 4e). Knockdown of MAVS by siRNA diminished NLS-RIG-I-mediated IFN $\beta$ promoter activation by NA reconstitution in $293 \mathrm{~T}$ cells (Fig. 4f) and IRF3 phosphorylation in NLS-RIG-I expressing A549 cells infected with PAfs $\triangle$ NS1 (Fig. 4g, lane 15 vs. lane 18). To further demonstrate the requirement of MAVS for nuclear RIG-I sensing, RIG-I-MAVS double KO (DKO) A549 cell line was generated followed by episomal complementation with NLS-RIG-I (Supplementary Fig. 9a). While RIG-I KO cells expressing NLS-RIG-I showed substantial IRF3 nuclear translocation upon PAfs $\triangle N S 1$ infection, ablation of MAVS abolished NLS-RIG-I-mediated IRF3 activation in RIG-I-MAVS DKO cells (Fig. 4h). Moreover, no apparent change in the subcellular localization of MAVS was observed in infected cells expressing compartment-specific RIG-I (Supplementary Fig. 9b). These results demonstrated that the signaling pathway for NLS-RIG-I is consistent with the canonical RIG-I signaling axis for viral RNA sensing and IFN induction.

Nuclear RIG-I elicits antiviral response restricting IAV. We next sought to assess the antiviral effect conferred by nuclear RIG-I. The $\Delta$ NS1-ms virus was chosen to monitor the antiviral response as it had a relatively complete viral life cycle unlike the PAfs $\triangle$ NS1 virus. At 14 h.p.i, $\Delta$ NS1-ms induced IFN $\beta$ and IP10 mRNA expression in RIG-I KO A549 cells expressing NLS-RIG-I, albeit to slightly lower levels than that in SLN-RIG-I and NESRIG-I-expressing cells (Fig. 5a, b). Furthermore, NLS-RIG-I impeded viral protein accumulation as efficiently as SLN-RIG-I

Fig. 3 Nuclear RIG-I senses nuclear viral replication during RNP reconstitution. a 293T cells were RNP reconstituted (PR8 NA segment) in the presence of GFP, SLN-RIG-I, or NLS-RIG-I for $24 \mathrm{~h}$ and were subjected to cellular fractionation. The levels of viral RNA species in the cytoplasmic (Cyto) and nuclear (Nuc) fractions were determined by strand-specific QRT-PCR. Fold change is expressed using the $\Delta \Delta \mathrm{Ct}$ method relative to the nuclear RNA fraction of the PB1a reconstitution. b RNP reconstituted 293T cells (PR8 M segment) were subjected to immunofluorescence for FLAG-RIG-I (green) and FISH for M VRNA (red) at 24 h.p.t. Nuclei were stained with DAPI (blue). Scale bar $=10 \mu \mathrm{m}$. c 293T cells were cotransfected with plasmids encoding GFP or various RIG-I constructs along with p125Luc and pTK-rLuc followed by transfection with a 19-bp 5'ppp-dsRNA. RLUs are expressed as fold change relative to the GFP group without RNA stimulation (lane 1). Expression levels of FLAG-RIG-I/2CARD and GFP were determined by immunoblotting. Significant differences between mock-transfected and dsRNA-transfected cells expressing GFP or either RIG-I constructs were determined by two-way ANOVA followed by Sidak post-test. d, e 293T cells were RNP reconstituted with a Pol I vector or each of the eight viral segments in the presence of GFP, SLN-RIG-I, or NLS-RIG-I for $24 \mathrm{~h}$. RLUs are expressed as fold change relative to the Pol I vector reconstitution with GFP. Significant differences between reconstitutions with viral segments and Pol I vector were determined by one-way ANOVA followed by Tukey post-test. $\mathbf{f} 293 \mathrm{~T}$ cells were RNP reconstituted (PR8 NA segment) using either inactive (PB1a), WT, transcription-defective (T-), or replication-defective (R-) polymerases in the presence of GFP, SLN-RIG-I, NES-RIG-I, or NLS-RIG-I for $24 \mathrm{~h}$. RLUs are expressed as fold change relative to the PB1a reconstitution in the presence of GFP. $\mathbf{g}$ The levels of viral RNA species in RNP reconstituted cells (f) were determined by strand-specific qRT-PCR and are expressed as percentage changes compared to the reconstitution using WT polymerase. Data are shown as mean \pm SD of three experiments. ${ }^{\star \star \star} p<0.001$; ${ }^{\star \star \star \star} p<0.0001$; n.s. not significant 
a
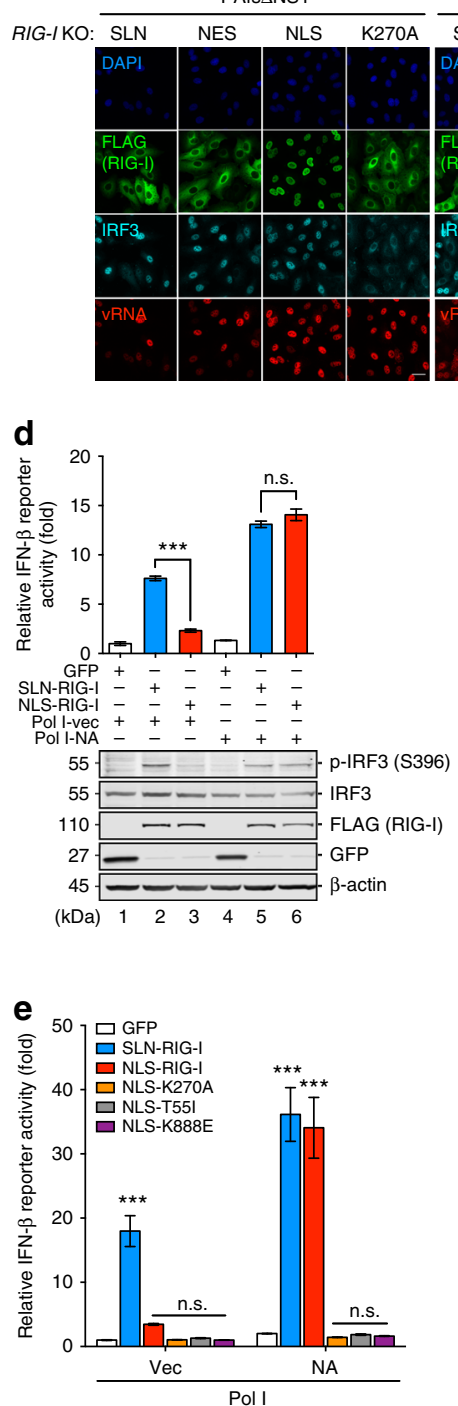

b
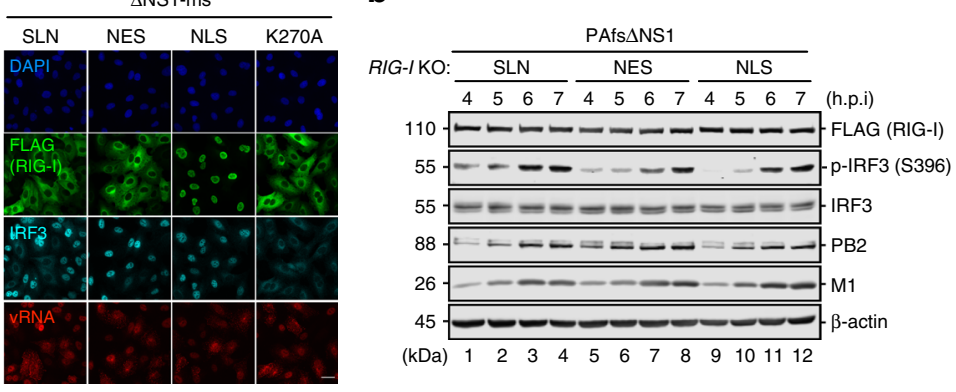

C

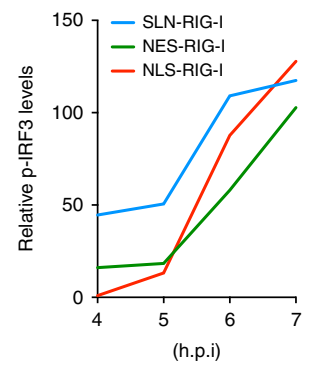

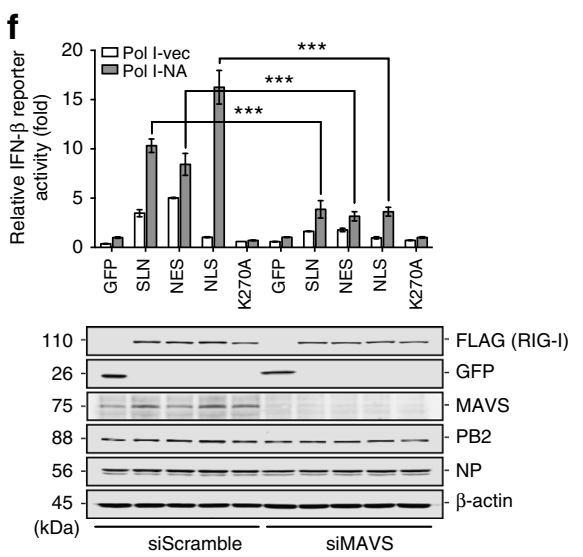
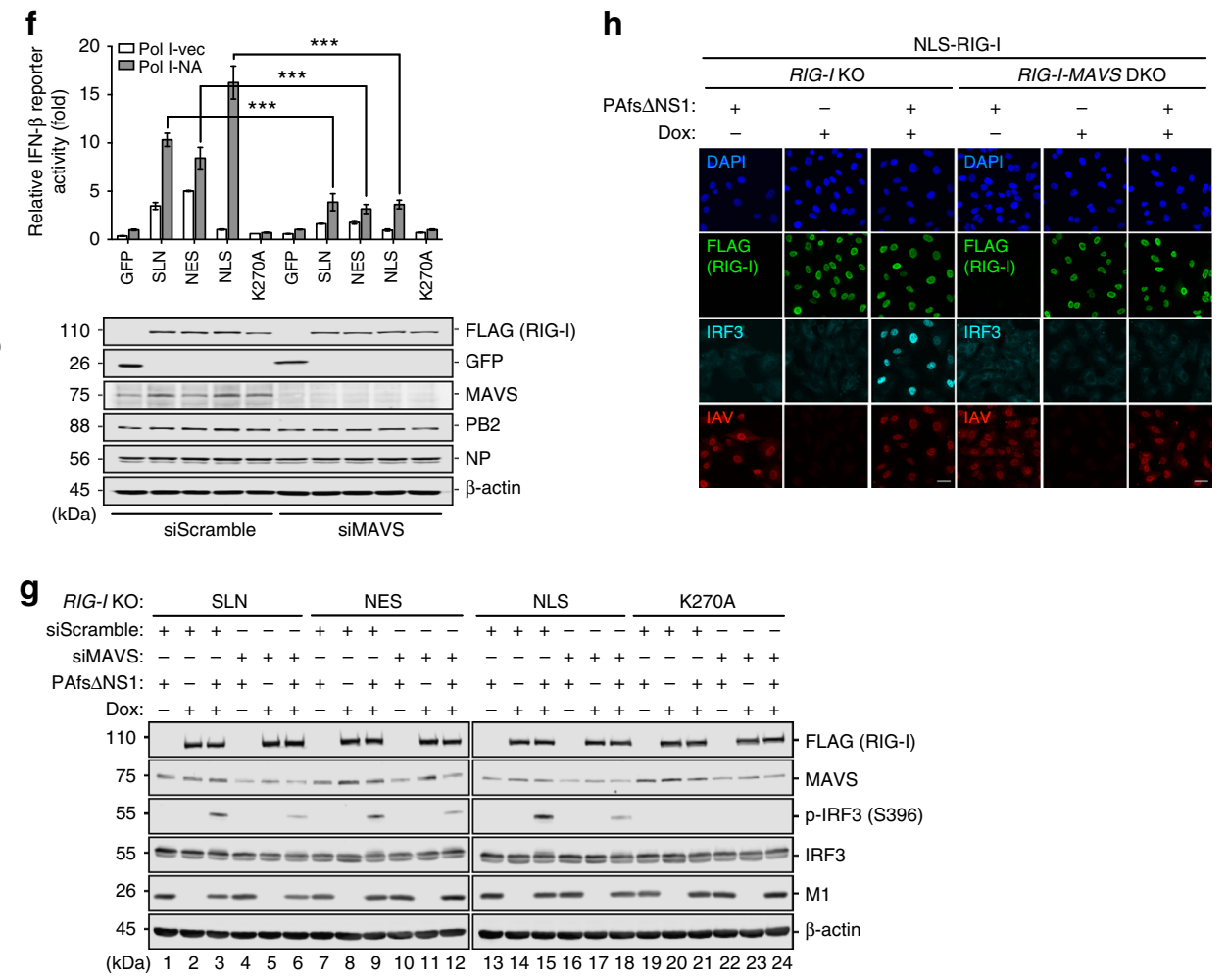

Fig. 4 Nuclear RIG-I senses IAV via the canonical signaling axis. a A549 RIG-I KO cell lines inducibly expressing SLN-RIG-I, NES-RIG-I, NLS-RIG-I, or K270A-RIG-I were induced with $1 \mu \mathrm{g} / \mathrm{mL}$ Dox for $4 \mathrm{~h}$ followed by infection with PAfs $\Delta$ NS1 or $\Delta$ NS1-ms (MOI $=10)$ for $7 \mathrm{~h}$. The cells were subjected to immunofluorescence for FLAG-RIG-I (green) and IRF3 (cyan), and FISH for M vRNA (red). b Dox-induced A549 cell lines were infected with PAfs $\Delta$ NS1 virus $(\mathrm{MOI}=10)$. At indicated h.p.i, the protein expression was determined by immunoblotting. $\mathbf{c}$ Kinetics of IRF3 phosphorylation (b) normalized to total IRF3 and $\beta$-actin levels were determined by densitometric analysis (ImageJ). d, e 293T cells were RNP reconstituted with Pol I vector or PR8 NA segment in the presence of GFP, SLN-RIG-I, or NLS-RIG-I (d), or in the presence of NLS-RIG-I harboring K270A, T55I, and K888E mutations (e) for $24 \mathrm{~h}$. The protein expression was determined by immunoblotting (d). RLUs are expressed as fold change relative to the Pol I vector reconstitution in the presence of GFP (d, e). $\mathbf{f} 293 \mathrm{~T}$ cells were transfected with $10 \mathrm{nM}$ scramble siRNA (siScramble) or MAVS siRNA (siMAVS) for $24 \mathrm{~h}$ followed by RNP reconstitution in the presence of GFP or various RIG-I constructs for another $24 \mathrm{~h}$. RLUs are expressed as that in $\mathbf{d}$ and $\mathbf{e}$. Data are shown as mean \pm SD of three experiments. Significant differences were determined by an unpaired Student's $t$-test (d) or one-way ANOVA followed by Tukey post-test (e, f). ${ }^{\star \star \star} p<0.001 ; n . s$. not significant. $\mathbf{g}$ Inducible A549 cell lines were transfected with $10 \mathrm{nM}$ indicated siRNA for $40 \mathrm{~h}$, followed by Dox induction ( $4 \mathrm{~h}$ ) and PAfs $\Delta$ NS1 infection $(\mathrm{MOI}=10,6 \mathrm{~h})$. The protein expression was determined by immunoblotting. $\mathbf{h}$ A549 RIG-I KO and RIG-I-MAVS DKO cell lines complemented with NLSRIG-I were left non-induced, or Dox-induced followed by mock or PAfs $\Delta$ NS1 infection $(\mathrm{MOI}=10,8 \mathrm{~h})$. Immunofluorescence was performed to detect FLAG-RIG-I (green), IRF3 (cyan), and IAV (red). Nuclei were stained with DAPI (blue). Scale bar $=25 \mu \mathrm{m}(\mathbf{a}, \mathbf{h})$

and NES-RIG-I at 24 h.p.i (Fig. 5c, lane 10 vs. lanes 2 and 6, compared to non-Dox induced lanes). NLS-RIG-I expression also led to reduced virus titer, albeit to a lesser extent compared to SLN-RIG-I and NES-RIG-I (Fig. 5d). No inhibitory effect on viral protein accumulation and titer was observed in RIG-I-MAVS DKO cells expressing either RIG-I (Fig. $5 c$, d). In line with previous studies $^{26,45}$, the signaling-inactive K270A mutant of RIG-I, albeit deficient in IFN-inducing capacity (Fig. 5a, b), consistently exhibited an antiviral effect to some extent. Such signalingindependent activity also required MAVS (Fig. 5c, d).
Collectively, these findings demonstrated that the nuclearresident RIG-I was able to restrict IAV replication by inducing a MAVS-dependent IFN response and antiviral immunity.

Nuclear RIG-I shows compartment-specific signaling capacity. We last assessed whether nuclear RIG-I confers signaling specificity toward viral agonists in the nucleus. To this end, we examined the ability of NLS-RIG-I to sense the cytoplasmicreplicating $\mathrm{SeV}$. As with the conditions for IAV infection 
a
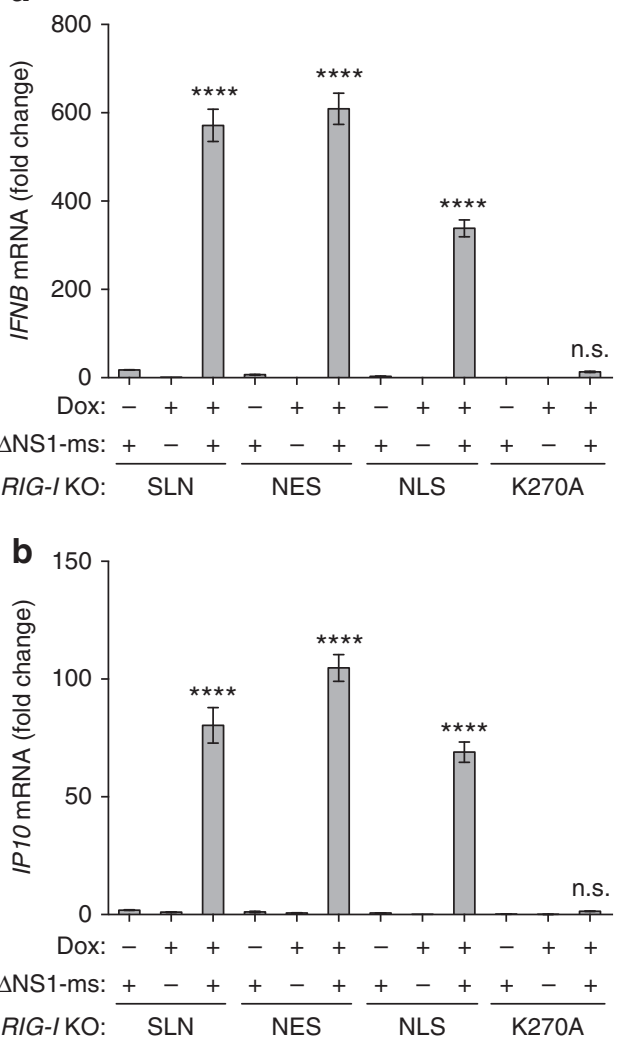

C

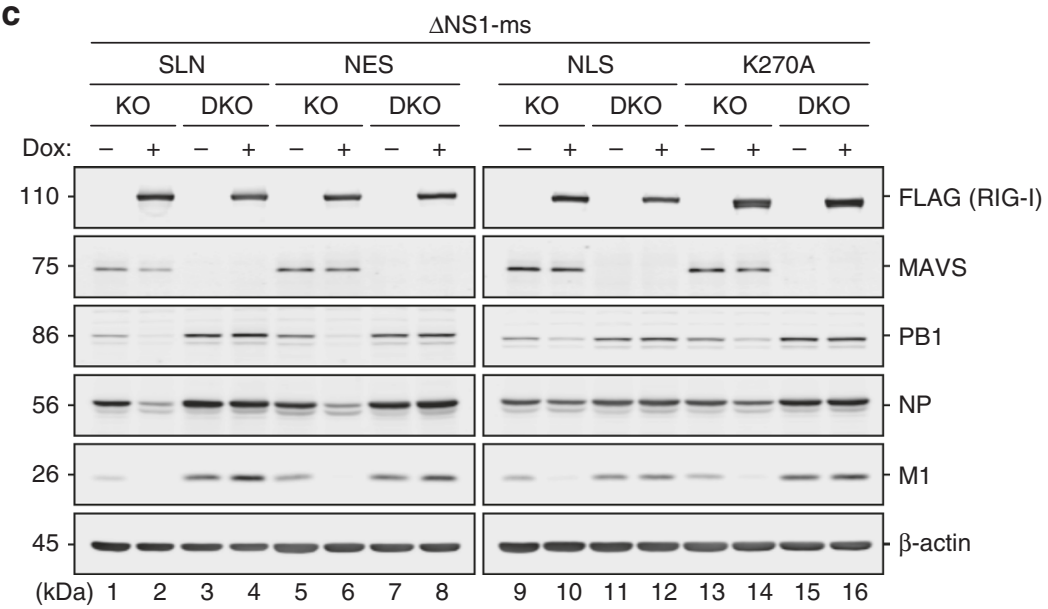

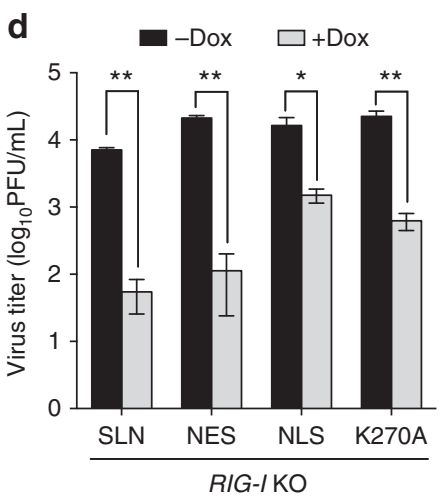

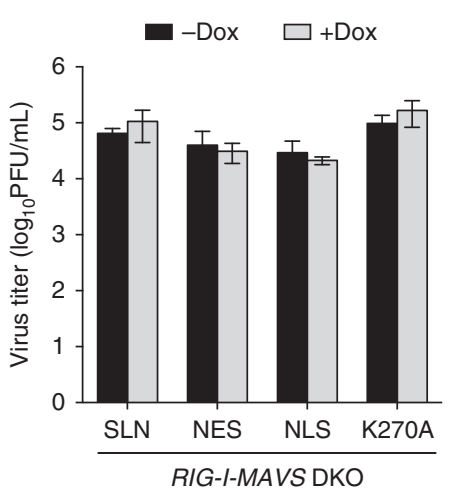

Fig. 5 Nuclear RIG-I restricts IAV infection. a, b A549 RIG-I KO cell lines inducibly expressing SLN-RIG-I, NES-RIG-I, NLS-RIG-I, or K270A-RIG-I were induced with $1 \mu \mathrm{g} / \mathrm{mL}$ Dox for $4 \mathrm{~h}$, followed by infection with $\triangle N S 1-\mathrm{ms}(\mathrm{MOI}=1)$ for $14 \mathrm{~h}$. The mRNA levels of IFN $\beta$ and IP10 (CXCL10) were determined by qRT-PCR. Relative mRNA expression was normalized to GAPDH mRNA levels and expressed using the $\Delta \Delta C \mathrm{Ct}$ method relative to the SLN (with Dox but without infection) condition. c Inducible A549 RIG-I KO or RIG-I-MAVS DKO cell lines were induced as in $\mathbf{a}$ and $\mathbf{b}$, and infected with $\Delta$ NS1-ms (MOI =0.1) for $24 \mathrm{~h}$. Expression levels of FLAG-RIG-I, MAVS, PB1, NP, and M1 were monitored by immunoblotting. $\mathbf{d}$ Virus titers in the culture supernatants from c were determined by plaque assay in MDCK-NS1 cells. Data are mean \pm SD of three independent experiments. Significant differences were determined by one-way ANOVA followed by Tukey post-test $(\mathbf{a}, \mathbf{b})$ or an unpaired Student's $t$-test $(\mathbf{d}) .{ }^{\star} p<0.05,{ }^{\star \star} p<0.01$, ${ }^{\star \star \star \star} p<0.0001$; n.s. not significant

(Fig. 4a), RIG-I KO A549 cell lines complemented with the compartment-specific or signaling-inactive RIG-I were infected with $\mathrm{SeV}$ and probed for IRF3 nuclear translocation. At 8 h.p.i, while $\mathrm{SeV}$ stimulated IRF3 activation in SLN-RIG-I and NESRIG-I-expressing cells, cells expressing NLS-RIG-I did not respond to SeV replication, resembling that in K270A-RIG-Iexpressing cells (Fig. 6a). This null responsiveness by NLS-RIG-I to $\mathrm{SeV}$ was further confirmed by quantifying the percentage of cells with the IRF3 nuclear translocation at the single-cell level (Fig. 6b), and IRF3 phosphorylation across the infected cell population (Fig. 6c). At physiologically comparable levels, NLSRIG-I also failed to sense SeV replication, whereas it efficiently induced antiviral gene expression in response to IAV infection (Supplementary Fig. 7d-g). Together with the efficient IRF3 activation upon IAV replication (Fig. 4a), these results demonstrated a compartment-specific functional sensing by nuclear RIG-I of nuclear-replicating, but not cytoplasmic-replicating, virus.

To further extend the compartment-specific role of nuclear RIG-I to the sensing of a nuclear-replicating DNA virus, we chose HBV since its pgRNA has been characterized as a RIG-I agonist ${ }^{46}$. During the HBV life cycle, pgRNA was transcribed from the viral covalently closed circular DNA in the nucleus followed by nuclear export for reverse transcription ${ }^{47}$. To explore the potential association of nuclear-resident RIG-I with pgRNA, stable Huh-7 cells harboring an HBV genome were subjected to cellular fractionation and the nuclear extract was immunoprecipitated with the RIG-I antibody. As observed in IAV infection, endogenous RIG-I remained at basal levels in HBV replicon cells (Fig. 6d and Supplementary Fig. 10a); however, the trace of nuclear-resident RIG-I efficiently associated with pgRNA as compared with the IgG control (Fig. 6e). Rather than type I IFN induction, HBV pgRNA sensing by RIG-I stimulated a type III IFN response ${ }^{46}$. In Huh-7.5 cells whose genome encodes a signaling-incompetent endogenous RIG-I ${ }^{48}$, ectopic expression of a WT RIG-I mediated efficient IFN $\lambda 1$ mRNA production in response to transfection with the $\mathrm{WT}$, but not the $\mathrm{HBX}$-deficient HBV genome (Fig. 6f, left). This differential IFN $\lambda 1$ induction correlated with the relative levels of pgRNA accumulated in transfected cells; the $\mathrm{HBx}$-deficient $\mathrm{HBV}$ genome produced significantly less pgRNA (Fig. 6f, right), consistent with a previous report ${ }^{49}$. We next assessed whether nuclear RIG-I activation by HBV pgRNA contributes to type III IFN induction. SLN-RIG-I and NES-RIG-I responded efficiently to pgRNA accumulation leading to IFN $\lambda 1$ promoter activation from 24 to 48 h.p.t (Fig. $6 \mathrm{~g}$ ) and ISRE promoter activation at 48 h.p.t in 293T cells (Supplementary Fig. 10b). In comparison, NLS-RIG-I exhibited minimal autonomous signaling activity but mediated noticeable IFN $\lambda 1$ and ISRE promoter activation in 293T cells transfected with the HBV genome (Fig. 6g and Supplementary Fig. 10b). Moreover, NLSRIG-I sensing of pgRNA required its RNA binding activity, as RIG-I with K888E mutation ${ }^{43,44}$ was diminished in IFN $\lambda 1$ and 
a

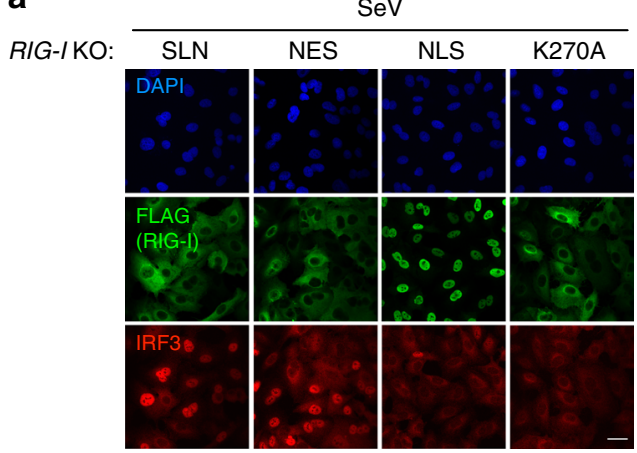

b

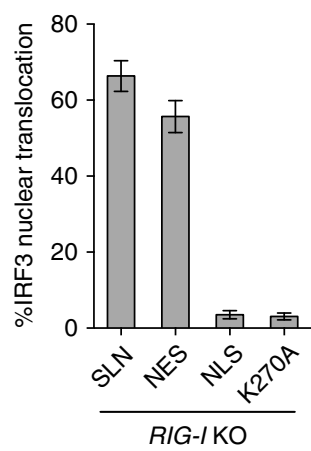

C

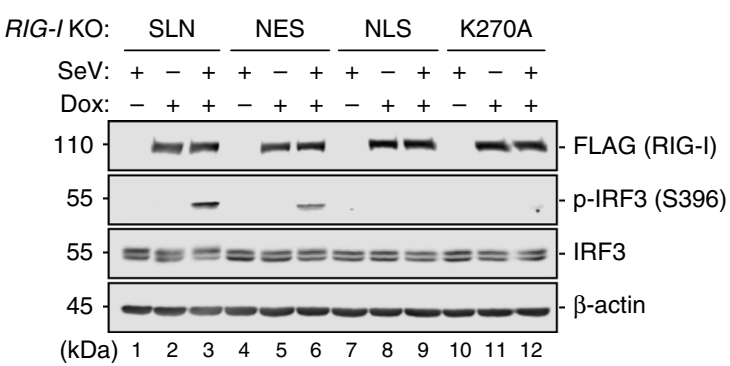

d

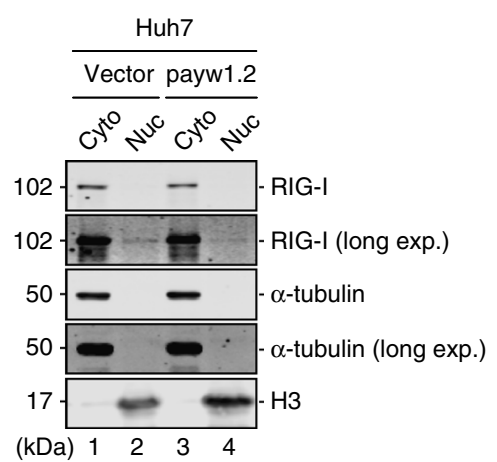

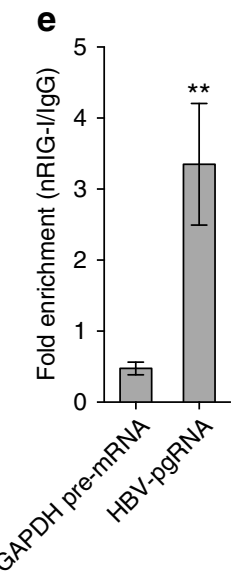

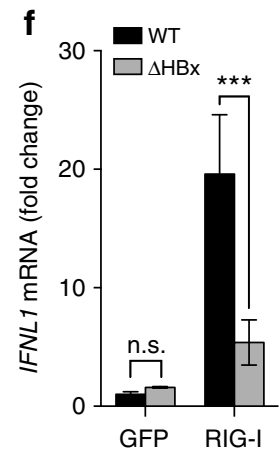

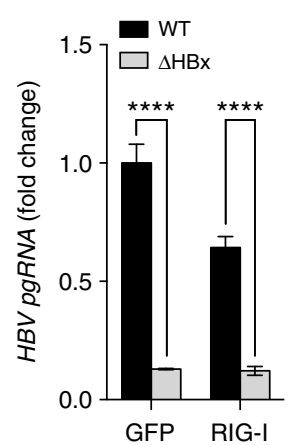

g

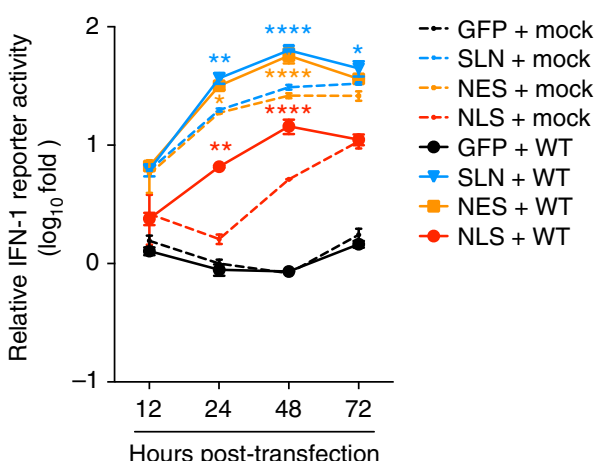

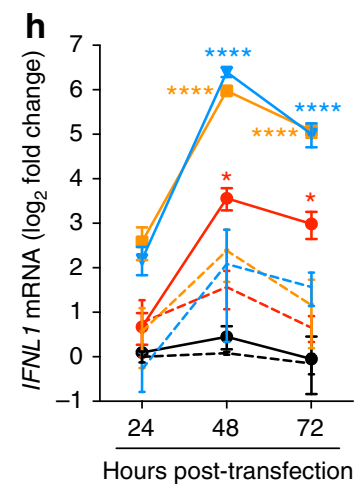

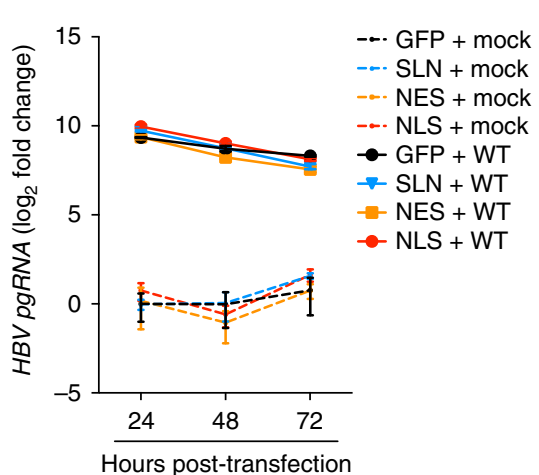

Fig. 6 Nuclear RIG-I exhibits compartment-specific signaling capacity. a A549 RIG-I KO cell lines inducibly expressing SLN-RIG-I, NES-RIG-I, NLS-RIG-I, or K270A-RIG-I were induced with $1 \mu \mathrm{g} / \mathrm{mL}$ Dox for $4 \mathrm{~h}$, followed by infection with SeV (50 HAU/mL) for $7 \mathrm{~h}$. Immunofluorescence was performed for FLAGRIG-I (green) and IRF3 (red). Nuclei were stained with DAPI (blue). Scale bar $=25 \mu \mathrm{m}$. $\mathbf{b}$ The percentage of cells positive for IRF3 nuclear translocation for each cell line (a) was calculated by quantifying ten random fields containing 300 RIG-I-positive cells. c Dox-induced A549 cell lines were infected with $\mathrm{SeV}$ as in a. The protein expression was determined by immunoblotting. $\mathbf{d}$ The presence of RIG-I in both the cytoplasmic (Cyto) and nuclear (Nuc) fractions of stable Huh-7 cell lines was determined by immunoblotting. e RIP was performed using the nuclear extracts of Huh-7-pawy1.2 cells. The levels of HBV pgRNA and GAPDH pre-mRNA in IgG or RIG-I immunoprecipitates were determined by qRT-PCR and are expressed as fold enrichment in the nuclear RIG-I immunoprecipitate relative to IgG. f Huh-7.5 cells were cotransfected with WT or $\triangle$ HBx HBV genome along with plasmids encoding GFP or RIG-I for $48 \mathrm{~h}$. The levels of IFN $\lambda 1$ mRNA and HBV pgRNA were determined by qRT-PCR. Fold change is expressed relative to the WT HBV transfection in the presence of GFP. $\mathbf{g} 293$ T cells were cotransfected with WT HBV genome along with plasmids encoding GFP or various RIG-I constructs, pGL-IFN $\lambda$ 1fLuc, and pTK-rLuc. RLUs at indicated h.p.t are expressed as fold change relative to the mock transfection in the presence of GFP at $12 \mathrm{~h}$. $\mathbf{h}$ Huh-7.5 cells were mock transfected or transfected with WT HBV genome in the presence of GFP or various RIG-I constructs. Kinetics of IFN $\lambda 1$ mRNA and HBV pgRNA expression were determined by qRT-PCR. Fold change is expressed relative to the mock transfection in the presence of GFP at $24 \mathrm{~h}$. Data are mean \pm SD of two independent experiments and significant differences were determined by an unpaired Student's $t$-test $(\mathbf{e}-\mathbf{h}) .{ }^{\star} p<0.05,{ }^{\star \star} p<0.01,{ }^{\star \star \star} p<0.001$, ${ }^{\star \star \star \star} p<0.0001 ;$ n.s. not significant

ISRE promoter activation (Supplementary Fig. 10b, c). Consistent with that in $293 \mathrm{~T}$ cells, NLS-RIG-I mediated IFN $\lambda 1$ mRNA production from 48 to 72 h.p.t in HBV-transfected Huh-7.5 cells, albeit to a lesser extent than SLN-RIG-I and NES-RIG-I (Fig. 6h).
Collectively, although the nuclear RIG-I was less potent than its cytoplasmic counterpart in pgRNA sensing and type III interferon induction, it was appreciably involved in sensing viral agonists derived from within the nucleus. 


\section{Discussion}

Our findings on nuclear-resident RIG-I provide advanced insight into the spatiotemporal detection of IAV by RIG-I, which has long been a puzzle given the nuclear replicating nature of IAV. To date, the time frame of RIG-I activation in the course of IAV infection has been attributed to at least three stages. These include RIG-I activation (1) by incoming vRNPs prior to nuclear import $^{25,26}$; (2) during the course of viral RNA synthesis in the nucleus ${ }^{29}$; and (3) by progeny vRNPs following their nuclear export $^{21}$. Among those, controversies remain regarding the relative contribution of the incoming vRNPs and the course of nuclear viral RNA synthesis to RIG-I activation. Although the incoming vRNPs immediately associate with RIG-I ${ }^{25,26}$, whether it leads to RIG-I activation and IFN induction remains unclear. In line with recent studies 29,50 , our data support the major contribution of nuclear viral RNA synthesis to RIG-I activation. Since RIG-I has been regarded as an exclusive cytoplasmic sensor, previous studies rationalized that the nuclear export of viral agonists is a prerequisite for RIG-I activation. Accordingly, this notion was supported by the observation that a chemical inhibitor causing nuclear retention of viral mRNA abolished IRF3 activation $^{29}$. However, while the nuclear retention of viral mRNA does not necessarily indicate that of other viral RNA species, treatment with the chemical inhibitor substantially diminished viral replication during which the essential agonists activating RIG-I are produced $^{21,24}$. Given the identification of nuclear RIG-I in our study, we propose a shifting paradigm in which the RNA nuclear export is dispensable for RIG-I activation. While the nuclear export of viral agonists is required for the activation of cytoplasmic RIG-I, the nuclear RIG-I could directly sense the nucleusderived viral agonists and initiate the onset of IFN induction. This additional layer of non-self surveillance in the nuclear compartment is particularly relevant for viruses that have a nuclear phase in their life cycles.

During the course of IAV infection, the relative contributions of the two RIG-I pools are distinct, which fit in the time frame of the nucleocytoplasmic trafficking of vRNP. Nuclear RIG-I accounts for the early association with vRNP while both RIG-I pools are involved in vRNP interaction with the progression of vRNP nuclear export. Although vRNP association indicates closer proximity to the immunostimulatory RNA species such as the genomic vRNA, the early capture of vRNP by nuclear RIG-I did not facilitate a more rapid signaling activation. Either pool of RIG-I by itself mediated a delayed activation of IRF3 during a single-cycle infection, suggesting a coordinated contribution by both the cytoplasmic and nuclear RIG-I to IAV sensing. Prior to the nuclear export of progeny vRNPs, while the cytoplasmic RIGI provided limited sensing of incoming vRNP and/or undefined viral RNA species exported from the nucleus during nuclear viral replication, the nuclear RIG-I maximized the sensing by 6 h.p.i where nuclear viral RNA synthesis occurred at maximum capacity. During HBV replication, the nuclear RIG-I associated with pgRNA in the nucleus but only modestly induced type III interferons compared with the cytoplasmic RIG-I. As the nuclear RNA synthesis and export mechanisms of HBV differ substantially from that of IAV, the exact mode of nuclear RIG-I activation by HBV merits further investigation.

Currently, no clear nucleocytoplasmic shuttling activity is defined for RIG-I. While a previous study proposed a RIG-I nuclear translocation model driven by its interaction with IAV vRNP components ${ }^{51}$, we demonstrated nuclear localization of RIG-I even in the absence of viral infection. Mechanistically, nuclear RIG-I activation appears to require its shuttling out of the nucleus, as all its downstream adapters, including TRIM25 and MAVS, are located in the cytoplasm. However, this translocation may not be absolutely required if a complete signaling cascade could be established within the nucleus. Interestingly, the recently identified nuclear TRIM25 fits into our finding, which would facilitate nuclear RIG-I activation ${ }^{52}$. Upon IAV infection, no noticeable change in MAVS localization was observed, suggesting there is no reorganization of MAVS to facilitate interaction with activated nuclear RIG-I. Previous studies reported that MAVS filaments exhibited intertwined network with perinuclear distribution, indicating a close association with nuclear membrane ${ }^{53,54}$. Such intimate contact and even fusion of mitochondria with nuclear membrane have long been observed to provide an essential gateway for energy delivery dictating cellular mRNA transport ${ }^{55}$. Thus, it is conceivable that the activated nuclear RIG-I interacts with the mitochondria and downstream adapters at the nuclear membrane interface at regions of greater permeability. Interestingly, IAV infection induces Nup153 nucleoporin degradation resulting in nuclear pore enlargement to passively facilitate vRNP export ${ }^{56}$. Therefore, we propose that during IAV infection, an altered nuclear membrane architecture may facilitate the formation of a sensing milieu for nuclear RIG-I at the perinuclear region (Supplementary Fig. 11). Such perinuclear membrane-associated pattern also emerges for cytoplasmic RIG-I during acute $\mathrm{SeV}$ infection ${ }^{53,57}$. Moreover, the requirement of compromised integrity of nuclear envelope has also been shown for herpesvirus-induced relocalization of host $5 \mathrm{~S}$ ribosomal RNA pseudogene transcripts activating RIG-I ${ }^{58}$.

Overall, the identification and characterization of nuclearresident RIG-I in relation to IAV infection provide the first evidence of a non-self RNA sensing paradigm in the nucleus. Compared to other members of the $\mathrm{DExD} / \mathrm{H}$-box RNA helicase family which are mostly nuclear shuttle or exclusively nuclear proteins ${ }^{59}$, the nuclear localization of RIG-I likely implies a remnant function retained in the nucleus during its evolution toward the cytoplasmic compartment. Here we define such function as the active sensing of nucleus-derived viral RNA contributing to antiviral immunity. Understanding the nuclear roles for other RLRs and RNA sensors would facilitate the establishment of a complete nuclear RNA sensing paradigm and the coordination of RNA sensing pathways from different cellular compartments culminating in virus restriction.

\section{Methods}

Cell culture. Madin-Darby canine kidney (MDCK, ATCC, \#CRL-2936) and human embryonic kidney (HEK293T, ATCC, \#CRL-3216) cells were maintained in minimal essential medium Eagle (MEM, Sigma) and Dulbecco's Modified Eagle's Medium (DMEM, Sigma) supplemented with $10 \%$ FBS and $50 \mu \mathrm{g} / \mathrm{mL}$ gentamicin, respectively. The MDCK cell line inducibly expressing the NS1 protein of A/Puerto Rico/8/34 (PR8) was constructed by lentiviral transduction of MDCK cells with a Tet-On inducible lentiviral vector containing the PR8 NS1 open reading frame (ORF). The MDCK-NS1 cell line was cultured in MEM supplemented with $10 \%$ tetracycline-free FBS (Clontech), $50 \mu \mathrm{g} / \mathrm{mL}$ gentamicin, and $5 \mu \mathrm{g} / \mathrm{mL}$ puromycin (Invivogen). Human lung epithelial (A549, ATCC, \#CRM-CCL-185) cells and its derivative knock-out (KO) cells were maintained in Ham's F-12K (Kaighn's) medium (Gibco) containing 10\% FBS and $50 \mu \mathrm{g} / \mathrm{mL}$ gentamicin. A549 RIG-I KO and RIG-I-MAVS DKO cells inducibly expressing scrambled tagged (SLN)-RIG-I, NES-RIG-I, NLS-RIG-I, and RIG-I with K270A mutation were cultured in F-12K with $10 \%$ tetracycline-free FBS, gentamicin, and $2 \mu \mathrm{g} / \mathrm{mL}$ puromycin. Human hepatoma Huh-7.5 cells and Huh-7 cells stably transfected with an empty vector or a greater-than-unit-length HBV genome (pawy1.2) carrying a luciferase reporter were maintained in DMEM supplemented with $10 \%$ FBS, with the stable cell lines additionally supplemented with $100 \mu \mathrm{g} / \mathrm{mL}$ hygromycin B (Invivogen). All cell cultures were maintained at $37^{\circ} \mathrm{C}$ in a humidified $5 \% \mathrm{CO}_{2}$ atmosphere and were monthly tested for mycoplasma contamination.

DNA constructs and transfection. The human RIG-I ORF was amplified from the pEF-flagRIG-I ${ }^{41}$ and subcloned into pCMV-3xFLAG. To generate compartment-specific RIG-I constructs, a monopartite SV40 large T antigen NLS (PKKKRKV) and a nuclear factor erythroid 2-related factor 2 NES

(LKKQLSTLYL) were inserted between the $3 \times$ FLAG tag and RIG-I ORF, respectively, giving rise to pCMV-3×FLAG-NLS-RIG-I and pCMV-3×FLAG-NES-RIG-I. In parallel, an inverted NLS sequence (NLPLFLR) was inserted at the same location and designated SLN-RIG-I as a scrambled control. Site-directed mutagenesis on 
pCMV-3×FLAG-NLS-RIG-I (T55I, K270A, and K888E) or on viral polymerases in the backbone of pcDNA3.1 (PB2-R142A, PB2-E361A, PB1-D445A/D446A, PA-

D108A, and PA-E410A) was introduced by overlapping PCR. Episomal constructs, with Tet-On inducible expression of $3 \times$ FLAG-SLN-RIG-I, NES-RIG-I, NLS-RIG-I, or K270A-RIG-I, were constructed by cloning the respective ORFs downstream of a minimal CMV promoter that is behind a tetracycline responsive element (TRE promoter). This vector also contains expression cassettes for the transactivator rtTA, puromycin resistance, and the Epstein-Barr virus nuclear antigen 1 (EBNA1). For RIG-I KO, an annealed oligonucleotide pair encoding the 20-nt guide sequences (GGGTCTTCCGGATATAATCC) targeting the exon 1 of human RIG-I was ligated in between the two BbsI sites of the CRISPR/Cas9 plasmid pSpCas9-2A-Puro (PX459) v2.0 (Addgene \#62988). The correct sequence of all constructs was confirmed by DNA sequencing (Eurofins). The sequences of cloning primers are listed in Supplementary Table 1. Transient transfection of 293T and Huh-7.5 cells was performed using TransIT-LT1 (Mirus Bio) and jetPEI (Polyplus) as per manufacturer's instructions, respectively.

Viruses and reverse genetics. The recombinant viruses were generated by the eight-plasmid reverse-genetics system as previously described with slight modification ${ }^{60}$. The set of $\mathrm{pHW}$ plasmids (pHW191-198) encoding the eight $\mathrm{A} /$ Puerto Rico/8/34 (H1N1) segments was used as the backbone for mutant virus generation. PA-X/NS1 double-deficient PR8 (PAfs $\triangle \mathrm{NS1}$ ) contains PA segment with abolished +1 ribosomal frameshifting for X-ORF translation ${ }^{34}$, and NS segment with the intron region deleted. Rescue of PA-X/NS1 double-deficient virus was performed in co-cultured MDCK-NS1/293T cells in Opti-MEM (Gibco) containing $0.2 \%$ bovine serum albumin (BSA), $1 \mu \mathrm{g} / \mathrm{mL}$ L-1-tosylamide-2-phenylethyl chloromethyl ketone (TPCK)-trypsin, and $1 \mu \mathrm{g} / \mathrm{mL}$ Dox (Sigma). The rescued virus was propagated and titrated in MDCK-NS1 cells. The genome authenticity of segments 3 and 8 of the mutant virus was verified by RT-PCR followed by DNA sequencing. The widely used PR8 $\triangle$ NS1 virus $(\triangle \mathrm{NS} 1-\mathrm{ms})^{33}$ was propagated in MDCK-NS1 cells. A/ Victoria/3/75 (H3N2) and a low pathogenic A/chicken/British Columbia/CN-6/ 2004 (H7N3) were propagated in MDCK cells and embryonated chicken eggs, respectively. SeV (Cantell strain) was obtained from ATCC (VR-907) and propagated in embryonated chicken eggs. All virus infections were performed at the indicated multiplicity of infection (MOI) by incubating the virus inoculum diluted in Dulbecco's Phosphate-Buffered Saline containing calcium and magnesium (DPBS $+/+$, Hyclone) with cells for $1 \mathrm{~h}$. The inoculum was then removed and replaced with plain medium containing $0.2 \%$ BSA and $1 \mu \mathrm{g} / \mathrm{mL}$ TPCK-trypsin (or $0.25 \mu \mathrm{g} / \mathrm{mL}$ for A549 cells and its derivatives). TPCK-trypsin was omitted from the culture medium for single-cycle infections.

A549 cells inducibly expressing compartment-specific RIG-I. The CRISPR Cas9 system was employed to generate RIG-I KO, MAVS KO, and RIG-I-MAVS DKO A549 cells ${ }^{61}$. Briefly, A549 cells were transfected with pSpCas9-2A-Puro (PX459) v2.0 plasmid harboring the sgRNA sequence using lipofectamine 3000 transfection reagent (Invitrogen) as per manufacturer's instructions. At $24 \mathrm{~h}$ posttransfection (h.p.t.), cells were subjected to puromycin selection at a concentration of $2 \mu \mathrm{g} / \mathrm{mL}$. Two days later ( $72 \mathrm{~h} . \mathrm{p.t}$ ), puromycin was removed from the culture medium to avoid genome integration of the CRISPR plasmid. After 2 weeks, monoclonal cell lines were isolated by limiting dilution at a density of 0.5 cells/well followed by clonal expansion for 3 weeks. Fourteen selected clones were characterized by examining RIG-I expression level induced by SeV infection (100 HAU/ $\mathrm{mL}$ ) and IFN $\beta 1$ a treatment (500 U/mL, IF014, Millipore). Genomic DNA was further extracted from two of the selected clones using a DNeasy Blood \& Tissue Kit (Qiagen) and amplified for the region flanking RIG-I exon 1. One monoclonal cell line (KO3) exhibited a homozygous single-nucleotide insertion within the sgRNA targeting site. To generate MAVS KO and RIG-I-MAVS DKO A549 cells, WT A549 or the KO3 cells were transfected with plasmids harboring three sgRNA sequences targeting human MAVS (Santa Cruz). Monoclonal cell lines were selected by limiting dilution and characterized by the ablation of MAVS expression. The monoclonal RIG-I KO (KO3) and RIG-I-MAVS DKO (DKO4) cell lines were further used to construct reconstituted A549 cell lines inducibly expressing compartment-specific RIG-I. To avoid any off-target effect conferred by lentiviral vector-based delivery of exogenous gene expression, we chose a non-integrating episomal expression approach to construct these cell lines. The KO3 and DKO4 cells were transfected with episomal plasmids expressing FLAG-tagged SLN-RIG-I, NES-RIG-I, NLS-RIG-I, or K270A-RIG-I under the control of a TRE promoter followed by selection with $2 \mu \mathrm{g} / \mathrm{mL}$ puromycin for 4 weeks. After the establishment of persistent episomal replication, the stable cell lines were characterized by examining FLAG-RIG-I expression and localization in the presence or absence of $1 \mu \mathrm{g} / \mathrm{mL}$ Dox.

Co-immunoprecipitation and immunoblotting. A549 or HEK293T cells were infected with indicated viruses or RNP reconstituted followed by crosslinking in PBS (-l-) containing $1 \mathrm{mM}$ dithiobis[succinimidyl propionate] (DSP, Sigma) for $30 \mathrm{~min}$ on ice. Cells were subjected to cellular fractionation as described elsewhere ${ }^{52}$ and the nuclear pellets were extracted at $4^{\circ} \mathrm{C}$ for 30 min with a buffer containing $50 \mathrm{mM}$ Tris- $\mathrm{HCl}$ (pH 7.4), $200 \mathrm{mM} \mathrm{NaCl}, 1 \%$ NP-40, 1 mM EDTA, $50 \mu \mathrm{g} / \mathrm{mL}$ RNase A, and $1 \times$ cOmplete protease inhibitors (Roche). The nuclear extracts were further pre-cleared with $20 \mu \mathrm{L}$ protein G Dynabeads (Invitrogen) at $4{ }^{\circ} \mathrm{C}$ for $30 \mathrm{~min}$. For co-immunoprecipitation, $35 \mu \mathrm{L}$ protein $\mathrm{G}$ Dynabeads were conjugated with $3 \mu \mathrm{g}$ of either mouse IgG1 isotype control (CST) or mouse antiRIG-I antibody (1C3, Millipore) at $4{ }^{\circ} \mathrm{C}$ for $1 \mathrm{~h}$. Pre-cleared lysates were then incubated with the conjugated beads at $4{ }^{\circ} \mathrm{C}$ for $16 \mathrm{~h}$. On the next day, the beads were washed four times with the wash buffer $(50 \mathrm{mM}$ Tris- $\mathrm{HCl}, \mathrm{pH} 7.4,150 \mathrm{mM}$ $\mathrm{NaCl}, 1 \% \mathrm{NP}-40,1 \mathrm{mM}$ EDTA) and eluted in $1 \times$ Laemmli buffer containing $355 \mathrm{mM} \beta$-mercaptoethanol. Immunoblotting was performed as previously described $^{24}$. Briefly, protein samples were resolved by sodium dodecyl sulfate polyacrylamide gel electrophoresis (SDS-PAGE), transferred onto nitrocellulose membranes, and probed with indicated primary antibodies including rabbit antiRIG-I (EPR18629, Abcam, 1:500), mouse anti-FLAG (M2, Sigma, 1:1000), rabbit anti-FLAG (CST, 1:1000), rabbit anti-Phospho-IRF3 (Ser396) (D6O1M, CST, 1:1000), rabbit anti-IRF3 (D6I4C, CST, 1:1000), mouse anti-GFP (4B10, CST, 1:1000), mouse anti- $\alpha$-tubulin (Abcam, 1:1000), mouse anti- $\beta$-actin (8H10D10, CST, 1:1000), rabbit anti-Lamin B1 (ab16048, Abcam, 1:1000), and rabbit antihistone H3 (D1H2, CST, 1:1000). Rabbit polyclonal antisera against IAV PB2, PA $\mathrm{NP}$, and M1 were raised in-house, and rabbit polyclonal antibody against IAV NS1 (PA5-32243, 1:500) was obtained from Pierce. For cellular fractionation, $\alpha$-tubulin and histone $\mathrm{H} 3$ were used as markers for the cytoplasmic and nuclear fractions, respectively. Following incubation with donkey anti-rabbit IRDye 680RD and donkey anti-mouse IRDye 800CW secondary antibodies (LI-COR), membranes were visualized with an Odyssey infrared imaging system (LI-COR). All uncropped immunoblots are presented in Supplementary Fig. 12.

RNA immunoprecipitation (RIP). For RIP, Huh-7-HBV-payw1.2-Luc cells were DSP-crosslinked and an aliquot was saved for input RNA extraction. The remaining cells were subjected to cellular fractionation. The nuclear pellets were extracted with a buffer containing $50 \mathrm{mM}$ Tris- $\mathrm{HCl}(\mathrm{pH} 7.4), 200 \mathrm{mM} \mathrm{NaCl}, 1 \%$ NP-40, $1 \mathrm{mM}$ EDTA, $100 \mu \mathrm{g} / \mathrm{mL}$ E. coli tRNA (Roche), $100 \mathrm{U} / \mathrm{mL}$ RNasin (Promega), and $1 \times$ EDTA-free protease inhibitors at $4{ }^{\circ} \mathrm{C}$ for $1 \mathrm{~h}$. The nuclear extracts were pre-cleared with protein G Dynabeads for $30 \mathrm{~min}$ and incubated with IgG1 or RIG-I antibody conjugated Dynabeads overnight at $4{ }^{\circ} \mathrm{C}$. The beads were extensively washed with the wash buffer containing $50 \mathrm{mM}$ Tris- $\mathrm{HCl}$ (pH 7.4), $150 \mathrm{mM}$ $\mathrm{NaCl}, 0.5 \% \mathrm{NP}-40,1 \mathrm{mM}$ EDTA, $100 \mu \mathrm{g} / \mathrm{mL}$ tRNA, and $40 \mathrm{U} / \mathrm{mL}$ RNasin, followed by proteinase $\mathrm{K}$ digestion in a buffer containing $20 \mathrm{mM}$ Tris- $\mathrm{HCl}(\mathrm{pH} 7.4)$, $100 \mathrm{mM} \mathrm{NaCl}, 2 \mathrm{mg} / \mathrm{mL}$ proteinase K (NEB), $1 \% \mathrm{NP}-40,0.1 \% \mathrm{SDS}, 100 \mathrm{U} / \mathrm{mL}$ RNasin, $100 \mu \mathrm{g} / \mathrm{mL}$ tRNA, and $1 \mathrm{mM}$ EDTA at $37^{\circ} \mathrm{C}$ for $1.5 \mathrm{~h}$. The immunoprecipitated and input RNA were extracted using the TRIzol Reagent (Ambion) as per manufacturer's instructions and were reverse transcribed using oligo(dT) and random primers with SuperScript IV Reverse Transcriptase (Invitrogen). The levels of HBV pgRNA in IgG1 and RIG-I immunoprecipitates were determined by qRTPCR using specific primers targeting the $\varepsilon$ regions of pgRNA as previously described ${ }^{46}$. The levels of GAPDH pre-mRNA in both immunoprecipitates were determined in parallel as a negative control. The amount of each immunoprecipitated RNA was normalized to that in the input RNA, and was expressed as fold enrichment in the nuclear RIG-I immunoprecipitate relative to the IgG1 control.

Immunofluorescence and RNA FISH. A549 or HEK293T cells were grown on poly-L-lysine-coated 8-well or 16-well chamber slides (Nunc), and infected or transfected as indicated. At indicated time points post-infection or post-transfection, cells were rinsed twice with DPBS $(+/+)$ and fixed with $4 \%$ formaldehyde (methanol-free) in DPBS $(+/+)$ for $10 \mathrm{~min}$ at room temperature (RT). The cells were subsequently permeabilized with $0.2 \%$ Triton X-100 for 5 min and blocked with $5 \%$ BSA in DPBS $(+/+)$ at RT for $1 \mathrm{~h}$. Following incubation with primary antibodies including goat anti-IAV (AB1074, Millipore, 1:2000), mouse anti-IAV NP (AA5H, AbD Serotec, 1:500), rabbit anti-IRF3 (D6I4C, CST, 1:400), mouse anti-RIG-I (1C3, Millipore, 1:250), mouse anti-FLAG (M2, Sigma, 1:500), mouse anti-MAVS (E-3, Santa Cruz, 1:200), goat anti-FLAG (ab1257, Abcam, 1:500), and rabbit anti- $\beta$-tubulin (9F3, CST, 1:100), the cells were washed and incubated with secondary antibodies diluted in 5\% BSA for $1 \mathrm{~h}$. Secondary antibodies used include donkey anti-mouse Alexa Fluor 488, donkey anti-rabbit Alexa Fluor 546, donkey anti-rabbit Alexa Fluor 594, and donkey anti-goat Alexa Fluor 633 (Invitrogen, 1:500). After nuclear staining with 4',6-diamidino-2-phenylindole (DAPI) for 5 min, the cells were washed four times with DPBS $(+/+)$, once with RNase-free water, and mounted in ProLong Gold antifade mountant (Invitrogen). The FISH analysis was performed as previously described with modifications ${ }^{62,63}$. Briefly, following secondary antibody staining, the cells were subjected to another fixation step with $4 \%$ formaldehyde in DPBS $(+/+)$ for $10 \mathrm{~min}$, washed once, and equilibrated with $2 \times$ SSC ( $300 \mathrm{mM}$ sodium chloride, $30 \mathrm{mM}$ sodium citrate) with $10 \%$ formamide for $10 \mathrm{~min}$. To detect IAV vRNA in infected or RNP-reconstituted cells, a pool of 39 probes against viral M segment was custom designed (Stellaris, Biosearch Technologies) and the cells were incubated with $125 \mathrm{nM}$ Quasar 670conjugated probes in a hybridization buffer $(10 \%$ dextran sulfate, $2 \mathrm{mM}$ vanadyl-ribonucleoside complex (VRC), $0.02 \%$ RNA-free BSA, $1 \mathrm{mg} / \mathrm{mL}$ E. coli tRNA, $2 \times$ SSC, and $10 \%$ formamide) in a humidified chamber at $28^{\circ} \mathrm{C}$ for $16 \mathrm{~h}$. On the next day, the cells were washed with $2 \times$ SSC containing $10 \%$ formamide and $2 \mathrm{mM}$ VRC, stained with DAPI, and mounted as described above. Confocal microscopy was carried out using a Leica TCS SP8 confocal microscope, equipped with a Diode $(405 \mathrm{~nm})$, an Argon $(458,476,488,496,514 \mathrm{~nm})$, a DPSS (561 nm), 
and a Helium/Neon $(633 \mathrm{~nm})$ lasers. Image processing, quantification, and colocalization analysis were performed using the Fiji version of ImageJ (NIH).

RNP reconstitution and luciferase reporter assay. The RNP reconstitution was performed as previously described ${ }^{24}$. To monitor viral polymerase activity in the presence of compartment-specific RIG-I, HEK293T cells were transfected with the set of plasmids encoding the vRNP components (pcDNA-PB2, pcDNA-PB1, pcDNA-PA, pcDNA-NP, and pHH21-NP-fLuc), a constitutively expressing Renilla luciferase reporter plasmid (pTK-rLuc), alongside with plasmids expressing various RIG-I (pCMV-3×FLAG-SLN/NES/NLS-RIG-I) or GFP. To measure IFN $\beta$ promoter activation upon RNP reconstitution, 293T cells were transfected with plasmids as above except that the pHH21-NP-fLuc was replaced by pHH21 plasmids encoding each of the authentic viral RNA (pHH21-PB2 to NS), and a human IFN $\beta$ promoter-driven luciferase reporter plasmid (p125Luc) was supplemented. Transcription-defective (PB2-E361A, PA-D108A) or replication-defective (PB2R142A, PA-E410A) polymerase constructs were also included in place of the WT polymerase to determine the requirement of viral transcription and replication for IFN $\beta$ promoter activation upon RNP reconstitution. The relative luciferase activity (RLU) was determined at 24 h.p.t using the Dual-Luciferase Reporter Assay System (Promega). To test the signaling competency of N-terminally tagged RIG-I constructs, 293T cells were transfected with various RIG-I plasmids or GFP together with p125Luc and pTK-rLuc for $6 \mathrm{~h}$. The cells were further left untreated or stimulated with a 19-bp 5'ppp-dsRNA (Invivogen) using Lipofectamine RNAiMAX (Invitrogen) for $18 \mathrm{~h}$. For IFN $\lambda 1$ promoter activation by HBV, 293T cells were transfected with WT HBV (payw1.2) or $\triangle \mathrm{HBx} H \mathrm{HBV}$ (payw1.2*7) genome ${ }^{64}$ along with pGL4.18- $\lambda 1(-1070 /+46)$ and pTK-rLuc in the presence of compartmentspecific RIG-I or GFP. The RLU was determined at the indicated time points after transfection.

Cellular fractionation, strand-specific qRT-PCR, and qRT-PCR. HEK293T cells were transfected with plasmids encoding vRNP components in conjunction with GFP or indicated RIG-I constructs for $24 \mathrm{~h}$. Reconstitution in the presence of a catalytically inactive $\mathrm{PB} 1$ subunit (PB1a, D445A/D446A) was performed in parallel to set the background RNA levels. Reconstituted cells were washed twice in PBS $(-/-)$ and resuspended in a hypotonic buffer containing $10 \mathrm{mM}$ HEPES (pH 7.4), $10 \mathrm{mM} \mathrm{KCl}, 1.5 \mathrm{mM} \mathrm{MgCl} 2,1 \mathrm{mM}$ DTT, $1 \times$ EDTA-free protease inhibitors, and $100 \mathrm{U} / \mathrm{mL}$ RNasin. After incubation on ice for $10 \mathrm{~min}$, NP40 was added to a final concentration of $0.5 \%$ and the cell membrane was disrupted by vortexing at high speed for $15 \mathrm{~s}$. The disrupted cells were immediately spun down at $3500 \times g$ for $10 \mathrm{~min}$ and the supernatant was saved as the cytoplasmic fraction. The pellet was washed once more with the hypotonic buffer and saved as the nuclear fraction. Both fractions were resuspended in the TRIzol Reagent and subjected to RNA extraction per manufacturer's instruction. Extracted RNA from both fractions were resuspended in equal volume of water and stored at $-80^{\circ} \mathrm{C}$. Strand-specific qRTPCR was performed as previously described with modification ${ }^{24}$. Briefly, $5 \%$ of both cytoplasmic and nuclear RNA fractions were reverse transcribed using tagged strand-specific primers (sequences available upon request) for viral RNA species or oligo(dT) plus random primer for total RNA. Fold change of viral RNA levels was normalized to the cytoplasmic GAPDH mRNA levels and expressed using the $\Delta \Delta \mathrm{Ct}$ method relative to the nuclear RNA fraction of the PB1a reconstitution. Successful cellular fractionation was determined by immunoblotting for Lamin B1 and $\alpha$-tubulin in the nuclear and cytoplasmic fractions, respectively. Additionally, the exclusive presence of GAPDH pre-mRNA in the nuclear, but not the cytoplasmic fraction, served as a control at the RNA level. To determine mRNA levels of IFNB1 and C-X-C motif chemokine 10 (CXCL10; IP10) induced by the $\triangle \mathrm{NS1}$ ms virus or IFNL1 induced by HBV replication, total RNA was extracted from infected or transfected cells using an RNeasy Plus Mini Kit (Qiagen) as per manufacturer's instructions. Reverse transcription was carried out using oligo(dT) and SuperScript IV Reverse Transcriptase. qPCR was performed using Power SYBR Green PCR Master Mix (Applied Biosystems) on a StepOnePlus Real-Time PCR System (Applied Biosystems). Relative mRNA expression was normalized to mRNA levels of housekeeping genes (GAPDH for IAV infection or GUSB for HBV genome transfection) and expressed using the $\Delta \Delta \mathrm{Ct}$ method relative to the conditions as indicated. The sequences of all qPCR primers are listed in Supplementary Table 2.

RNA interference. The Silencer Select siRNA negative control and siRNA against human MAVS (sense: 5'-GGGUUCUUCUGAGAUUGAAtt-3'; antisense: 5'-UUCAAUCUCAGAAGAACCCag-3') were obtained from Invitrogen. Briefly, HEK293T and inducible A549 cells were transfected with $10 \mathrm{nM}$ siRNAs using Lipofectamine RNAiMAX (Invitrogen) and TransIT-X2 reagents (Mirus Bio), respectively. At 24 or $40 \mathrm{~h}$ post-transfection, cells were reconstituted with vRNP or infected with the mutant virus as indicated.

Statistical analysis. The statistical significance of differences was calculated using GraphPad Prism 7 (GraphPad Software, Inc., USA) with an unpaired Student's $t$-test, one-way or two-way ANOVA followed by the Tukey or Sidak post-hoc test to obtain the $p$-value. Data are shown as mean \pm SD of three independent experiments performed in triplicates unless otherwise indicated. Significant differences between groups are denoted by ${ }^{*} p<0.05,{ }^{* *} p<0.01$, ${ }^{* *} p<0.001$, or ${ }_{* * * *} p<0.0001$

Data availability. All relevant data are available upon request from the corresponding author.

Received: 14 March 2018 Accepted: 24 July 2018

Published online: 10 August 2018

\section{References}

1. Iwasaki, A. \& Medzhitov, R. Control of adaptive immunity by the innate immune system. Nat. Immunol. 16, 343-353 (2015).

2. $\mathrm{Wu}, \mathrm{J}$. \& Chen, Z. J. Innate immune sensing and signaling of cytosolic nucleic acids. Annu. Rev. Immunol. 32, 461-488 (2014).

3. Akira, S., Uematsu, S. \& Takeuchi, O. Pathogen recognition and innate immunity. Cell 124, 783-801 (2006).

4. Diner, B. A., Lum, K. K. \& Cristea, I. M. The emerging role of nuclear viral DNA sensors. J. Biol. Chem. 290, 26412-26421 (2015).

5. Orzalli, M. H., DeLuca, N. A. \& Knipe, D. M. Nuclear IFI16 induction of IRF3 signaling during herpesviral infection and degradation of IFI16 by the viral ICP0 protein. Proc. Natl Acad. Sci. USA 109, E3008-E3017 (2012).

6. Kerur, N. et al. IFI16 acts as a nuclear pathogen sensor to induce the inflammasome in response to Kaposi sarcoma-associated herpesvirus infection. Cell Host Microbe 9, 363-375 (2011).

7. Dutta, D. et al. BRCA1 regulates IFI16 mediated nuclear innate sensing of herpes viral DNA and subsequent induction of the innate inflammasome and interferon-beta responses. PLoS Pathog. 11, e1005030 (2015).

8. Jonsson, K. L. et al. IFI16 is required for DNA sensing in human macrophages by promoting production and function of cGAMP. Nat. Commun. 8, 14391 (2017).

9. Schlee, M. \& Hartmann, G. Discriminating self from non-self in nucleic acid sensing. Nat. Rev. Immunol. 16, 566-580 (2016).

10. Kato, H. et al. Differential roles of MDA5 and RIG-I helicases in the recognition of RNA viruses. Nature 441, 101-105 (2006).

11. Loo, Y. M. et al. Distinct RIG-I and MDA5 signaling by RNA viruses in innate immunity. J. Virol. 82, 335-345 (2008).

12. Shaw, M. L. \& Palese, P. in Fields Virology 6th edn (eds Knipe, D. M. \& Howley, P. M.) (Lippincott Williams \& Wilkins, Philadelphia, 2013).

13. Chan, Y. K. \& Gack, M. U. Viral evasion of intracellular DNA and RNA sensing. Nat. Rev. Microbiol. 14, 360-373 (2016).

14. Garcia-Sastre, A. Ten strategies of interferon evasion by viruses. Cell Host Microbe 22, 176-184 (2017).

15. Overby, A. K., Popov, V. L., Niedrig, M. \& Weber, F. Tick-borne encephalitis virus delays interferon induction and hides its double-stranded RNA in intracellular membrane vesicles. J. Virol. 84, 8470-8483 (2010).

16. Neufeldt, C. J. et al. The hepatitis $C$ virus-induced membranous web and associated nuclear transport machinery limit access of pattern recognition receptors to viral replication sites. PLoS Pathog. 12, e1005428 (2016).

17. Hale, B. G., Albrecht, R. A. \& Garcia-Sastre, A. Innate immune evasion strategies of influenza viruses. Future Microbiol. 5, 23-41 (2010).

18. Killip, M. J., Fodor, E. \& Randall, R. E. Influenza virus activation of the interferon system. Virus Res. 209, 11-22 (2015).

19. Josset, L., Frobert, E. \& Rosa-Calatrava, M. Influenza A replication and host nuclear compartments: many changes and many questions. J. Clin. Virol. 43, 381-390 (2008)

20. Opitz, B. et al. IFNbeta induction by influenza A virus is mediated by RIG-I which is regulated by the viral NS1 protein. Cell Microbiol. 9, 930-938 (2007).

21. Rehwinkel, J. et al. RIG-I detects viral genomic RNA during negative-strand RNA virus infection. Cell 140, 397-408 (2010).

22. Schlee, M. et al. Recognition of $5^{\prime}$ triphosphate by RIG-I helicase requires short blunt double-stranded RNA as contained in panhandle of negativestrand virus. Immunity 31, 25-34 (2009).

23. Baum, A., Sachidanandam, R. \& Garcia-Sastre, A. Preference of RIG-I for short viral RNA molecules in infected cells revealed by next-generation sequencing. Proc. Natl Acad. Sci. USA 107, 16303-16308 (2010).

24. Liu, G., Park, H. S., Pyo, H. M., Liu, Q. \& Zhou, Y. Influenza A virus panhandle structure is directly involved in RIG-I activation and interferon induction. J. Virol. 89, 6067-6079 (2015).

25. Liedmann, S. et al. Viral suppressors of the RIG-I-mediated interferon response are pre-packaged in influenza virions. Nat. Commun. 5, 5645 (2014).

26. Weber, M. et al. Influenza virus adaptation PB2-627K modulates nucleocapsid inhibition by the pathogen sensor RIG-I. Cell Host Microbe 17, 309-319 (2015).

27. Osterlund, P. et al. Incoming influenza A virus evades early host recognition, while influenza B virus induces interferon expression directly upon entry. J. Virol. 86, 11183-11193 (2012). 
28. Makela, S. M. et al. RIG-I signaling is essential for influenza B virus-induced rapid interferon gene expression. J. Virol. 89, 12014-12025 (2015).

29. Killip, M. J., Smith, M., Jackson, D. \& Randall, R. E. Activation of the interferon induction cascade by influenza a viruses requires viral RNA synthesis and nuclear export. J. Virol. 88, 3942-3952 (2014).

30. Shaw, A. E. et al. Fundamental properties of the mammalian innate immune system revealed by multispecies comparison of type I interferon responses. PLoS Biol. 15, e2004086 (2017).

31. Li, W. et al. Nrf2 possesses a redox-insensitive nuclear export signal overlapping with the leucine zipper motif. J. Biol. Chem. 280, 28430-28438 (2005).

32. Garcia-Sastre, A. Induction and evasion of type I interferon responses by influenza viruses. Virus Res. 162, 12-18 (2011).

33. Garcia-Sastre, A. et al. Influenza A virus lacking the NS1 gene replicates in interferon-deficient systems. Virology 252, 324-330 (1998).

34. Jagger, B. W. et al. An overlapping protein-coding region in influenza A virus segment 3 modulates the host response. Science 337, 199-204 (2012).

35. Pleschka, S. et al. A plasmid-based reverse genetics system for influenza A virus. J. Virol. 70, 4188-4192 (1996).

36. Biswas, S. K. \& Nayak, D. P. Mutational analysis of the conserved motifs of influenza A virus polymerase basic protein 1. J. Virol. 68, 1819-1826 (1994).

37. Hara, K., Schmidt, F. I., Crow, M. \& Brownlee, G. G. Amino acid residues in the N-terminal region of the PA subunit of influenza A virus RNA polymerase play a critical role in protein stability, endonuclease activity, cap binding, and virion RNA promoter binding. J. Virol. 80, 7789-7798 (2006).

38. Jorba, N., Coloma, R. \& Ortin, J. Genetic trans-complementation establishes a new model for influenza virus RNA transcription and replication. PLoS Pathog. 5, e1000462 (2009).

39. Gastaminza, P., Perales, B., Falcon, A. M. \& Ortin, J. Mutations in the Nterminal region of influenza virus $\mathrm{PB} 2$ protein affect virus RNA replication but not transcription. J. Virol. 77, 5098-5108 (2003).

40. Fodor, E. et al. A single amino acid mutation in the PA subunit of the influenza virus RNA polymerase inhibits endonucleolytic cleavage of capped RNAs. J. Virol. 76, 8989-9001 (2002).

41. Yoneyama, M. et al. The RNA helicase RIG-I has an essential function in double-stranded RNA-induced innate antiviral responses. Nat. Immunol. 5, 730-737 (2004).

42. Gack, M. U. et al. Roles of RIG-I N-terminal tandem CARD and splice variant in TRIM25-mediated antiviral signal transduction. Proc. Natl Acad. Sci. USA 105, 16743-16748 (2008).

43. Cui, S. et al. The C-terminal regulatory domain is the RNA $5^{\prime}$-triphosphate sensor of RIG-I. Mol. Cell 29, 169-179 (2008).

44. Takahasi, K. et al. Nonself RNA-sensing mechanism of RIG-I helicase and activation of antiviral immune responses. Mol. Cell 29, 428-440 (2008).

45. Yao, H. et al. ATP-dependent effector-like functions of RIG-I-like receptors. Mol. Cell 58, 541-548 (2015).

46. Sato, $\mathrm{S}$. et al. The RNA sensor RIG-I dually functions as an innate sensor and direct antiviral factor for hepatitis B virus. Immunity 42, 123-132 (2015).

47. Beck, J. \& Nassal, M. Hepatitis B virus replication. World J. Gastroenterol. 13 , 48-64 (2007)

48. Sumpter, R. Jr. et al. Regulating intracellular antiviral defense and permissiveness to hepatitis $\mathrm{C}$ virus RNA replication through a cellular RNA helicase, RIG-I. J. Virol. 79, 2689-2699 (2005).

49. Belloni, L. et al. Nuclear HBx binds the HBV minichromosome and modifies the epigenetic regulation of cccDNA function. Proc. Natl Acad. Sci. USA 106, 19975-19979 (2009).

50. Killip, M. J., Jackson, D., Perez-Cidoncha, M., Fodor, E. \& Randall, R. E. Single-cell studies of IFN-beta promoter activation by wild-type and NS1defective influenza A viruses. J. Gen. Virol. 98, 357-363 (2017).

51. Li, W., Chen, H., Sutton, T., Obadan, A. \& Perez, D. R. Interactions between the influenza A virus RNA polymerase components and retinoic acidinducible gene I. J. Virol. 88, 10432-10447 (2014).

52. Meyerson, N. R. et al. Nuclear TRIM25 specifically targets influenza virus ribonucleoproteins to block the onset of RNA chain elongation. Cell Host Microbe 22, 627-638 e627 (2017).

53. Sanchez-Aparicio, M. T., Ayllon, J., Leo-Macias, A., Wolff, T. \& Garcia-Sastre, A. Subcellular localizations of RIG-I, TRIM25, and MAVS complexes. J. Virol. 91, e01155-16 (2017).

54. Lin, R. et al. Dissociation of a MAVS/IPS-1/VISA/Cardif-IKKepsilon molecular complex from the mitochondrial outer membrane by hepatitis $\mathrm{C}$ virus NS3-4A proteolytic cleavage. J. Virol. 80, 6072-6083 (2006).

55. Prachar, J. Intimate contacts of mitochondria with nuclear envelope as a potential energy gateway for nucleo-cytoplasmic mRNA transport. Gen. Physiol. Biophys. 22, 525-534 (2003).
56. Muhlbauer, D. et al. Influenza virus-induced caspase-dependent enlargement of nuclear pores promotes nuclear export of viral ribonucleoprotein complexes. J. Virol. 89, 6009-6021 (2015).

57. Liu, H. M. et al. The mitochondrial targeting chaperone 14-3-3epsilon regulates a RIG-I translocon that mediates membrane association and innate antiviral immunity. Cell Host Microbe 11, 528-537 (2012).

58. Chiang, J. J. et al. Viral unmasking of cellular $5 \mathrm{~S}$ rRNA pseudogene transcripts induces RIG-I-mediated immunity. Nat. Immunol. 19, 53-62 (2018).

59. Fuller-Pace, F. V. DExD/H box RNA helicases: multifunctional proteins with important roles in transcriptional regulation. Nucleic Acids Res. 34, 4206-4215 (2006).

60. Hoffmann, E., Neumann, G., Kawaoka, Y., Hobom, G. \& Webster, R. G. A. DNA transfection system for generation of influenza A virus from eight plasmids. Proc. Natl Acad. Sci. USA 97, 6108-6113 (2000).

61. Ran, F. A. et al. Genome engineering using the CRISPR-Cas9 system. Nat. Protoc. 8, 2281-2308 (2013).

62. Chou, Y. Y. et al. Colocalization of different influenza viral RNA segments in the cytoplasm before viral budding as shown by single-molecule sensitivity FISH analysis. PLoS Pathog. 9, e1003358 (2013).

63. Thulasi Raman, S. N. et al. DDX3 interacts with influenza A virus NS1 and NP proteins and exerts antiviral function through regulation of stress granule formation. J. Virol. 90, 3661-3675 (2016).

64. Wu, Q. \& Liu, Q. HBx truncation mutants differentially modulate SREBP-1a and -1c transcription and HBV replication. Virus Res. 210, 46-53 (2015).

\section{Acknowledgements}

We thank S. Pleschka (Justus-Liebig University Giessen) for pMP plasmids harboring A/ Victoria/3/75 (H3N2) segments, T. Fujita (Kyoto University) for pEF-flagRIG-I plasmid, F. Zhang (Broad Institute) for pSpCas9(BB)-2A-Puro (PX459) v2.0 plasmid, B. L. Slagle (Baylor College of Medicine) for payw1.2 and payw1.2 7 plasmids, T. Kameyama (Hokkaido University) for pGL4.18- $\lambda 1(-1070 /+46)$ plasmid, and A. García-Sastre (Icahn School of Medicine at Mount Sinai) for the $\Delta$ NS1-ms virus. G.L. is partially supported by the Vaccinology and Immunotherapeutics (V\&I) Scholarship from the School of Public Health, University of Saskatchewan. This work was supported by a NSERC grant to Y.Z.

\section{Author contributions}

G.L. and Y.Z. conceived the project, designed experiments, and wrote the manuscript. G.L. performed all aspects of the experiments unless otherwise indicated. Y.L. performed DI detection and assisted with virus titration, immunofluorescence, and luciferase reporter assays. S.N.T.R. assisted with immunofluorescence in HeLa cells. F.X., Q.W., Z. L., R.B., and Q.L. provided reagents and resources. All authors discussed data and commented on the manuscript.

\section{Additional information}

Supplementary Information accompanies this paper at https://doi.org/10.1038/s41467018-05745-w.

Competing interests: The authors declare no competing interests.

Reprints and permission information is available online at http://npg.nature.com/ reprintsandpermissions/

Publisher's note: Springer Nature remains neutral with regard to jurisdictional claims in published maps and institutional affiliations.

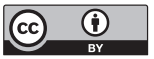

Open Access This article is licensed under a Creative Commons Attribution 4.0 International License, which permits use, sharing, adaptation, distribution and reproduction in any medium or format, as long as you give appropriate credit to the original author(s) and the source, provide a link to the Creative Commons license, and indicate if changes were made. The images or other third party material in this article are included in the article's Creative Commons license, unless indicated otherwise in a credit line to the material. If material is not included in the article's Creative Commons license and your intended use is not permitted by statutory regulation or exceeds the permitted use, you will need to obtain permission directly from the copyright holder. To view a copy of this license, visit http://creativecommons.org/ licenses/by/4.0/.

(C) The Author(s) 2018 